\title{
An Optimal Finite Element Method with Uzawa Iteration for Stokes Equations including Corner Singularities
}

\author{
Jae-Hong Pyo iD $^{1}$ and Deok-Kyu Jang (iD $^{2}$ \\ ${ }^{1}$ Department of Mathematics, Kangwon National University, Chuncheon 24341, Republic of Korea \\ ${ }^{2}$ Department of Applied Mathematics, Kyung Hee University, Yongin 17104, Republic of Korea
}

Correspondence should be addressed to Deok-Kyu Jang; dkjang@khu.ac.kr

Received 24 February 2021; Accepted 17 May 2021; Published 9 June 2021

Academic Editor: Xiaolong Qin

Copyright (c) 2021 Jae-Hong Pyo and Deok-Kyu Jang. This is an open access article distributed under the Creative Commons Attribution License, which permits unrestricted use, distribution, and reproduction in any medium, provided the original work is properly cited.

\begin{abstract}
The Uzawa method is an iterative approach to find approximated solutions to the Stokes equations. This method solves velocity variables involving augmented Lagrangian operator and then updates pressure variable by Richardson update. In this paper, we construct a new version of the Uzawa method to find optimal numerical solutions of the Stokes equations including corner singularities. The proposed method is based on the dual singular function method which was developed for elliptic boundary value problems. We estimate the solvability of the proposed formulation and special orthogonality form for two singular functions. Numerical convergence tests are presented to verify our assertion.
\end{abstract}

\section{Introduction}

To study the solution of a partial differential equation, the equation is sometimes interpreted in a weak (variational) sense and we can define the regular problem in this manner. Consider a variational problem:

$$
a(u, v)=(f, v)_{L^{2}}, \quad \text { for all } v \in V,
$$

where $H_{0}^{m} \subset V \subset H^{m}(\Omega)$ and $a(\cdot, \cdot)$ is bilinear form with continuity and coercivity. We call the problem is $H^{s}$-regular if, for every $f \in H^{s-2 m}(\Omega)$, there is a solution $u \in H^{s}(\Omega)$ with a constant $c$ such that

$$
\left\|u_{s}\right\| \leq c\|f\|_{s-2 m} .
$$

It is well known that the approximated solution of regular problems shows optimal convergence by using common numerical methods such as the finite difference method or the finite element method.

However, if a computational domain of an elliptic boundary value problem is a polygon including reentrant corners, then the problem is not $H^{s}$-regular, $s \geq 2$, and it is sometimes called a corner singular problem, and the singular solution is hard to approximate optimally. Therefore, there have been many numerical approaches developed to solve the problem efficiently. These methods aim to improve accuracy and to resolve the convergence difficulties. The $h p$ version of the finite element method is a typical method. It employs elements of variable sizes $h$ and polynomial degrees $p$ to improve the convergence rate and accuracy in [1].

The postprocessing method has been proposed in [2-6]. These methods calculate the coefficients of the singular coefficients from the finite element solution. Singular function boundary integral method also has been proposed to treat singular problems in [7-9]. These methods calculate the unknown coefficients of singular functions directly. The solution is approximated by the leadinig terms of the local asymptotic solution expansion, and the Dirichlet boundary conditions are weakly enforced utilizing Lagrange multipliers.

It is well known that the solution of an elliptic boundary value problem can be decomposed of a finite number of socalled singular functions, which come from the neighborhood of a corner point, and a smooth remainder function which is called a regular solution (see, for instance, [10-12]). The singular function method is one approach to use this fact. It takes into account the form of the singular solution by 
finding stress intensity factors and regular solution (see, for example, [13-15]). These methods consist of augmenting the spline space by the singular functions. The dual singular function is based on the observation that the regular part of the solution and the stress intensity factor are related to each other by testing a dual singular function in [16-18]. It was implemented as an iterative procedure that iterates back and forth between these equations. Also, this approach was extended to multigrid versions.

In [19], Cai and Kim developed and analyzed a finite element method for the accurate computation of the solution and intensity factors. If a regular part of the solution is smoother than the solution itself, then approximated solutions of a standard finite element lose accuracy. Therefore, the new method finds approximated regular solution first and then computes stress intensity factors and solution. This method decoupled variational formulations by testing the dual singular function and disjoint cut-off function. Consequently, the regular solution is uniquely determined by a well-posed variational problem, and stress intensity factors can be expressed by a regular solution. We call this method the finite element dual singular function method (FEDSFM).

This method was extended to other problems. Poisson problems with mixed boundary conditions and interface problems were applied in $[20,21]$. Also, this algorithm was studied to corner singularities of the Helmholtz equation and heat equation (see $[22,23])$. Some studies have been designed to target the singular solution of the Stokes equation which is much more complicated. We refer the interested reader to the papers $[24,25]$ for a formula for corner singularity of the Stokes equations. A mixed finite element method-based FE-DSFM was developed for Stokes equations in $[26,27]$. These proved the accuracy and wellposedness of the algorithm including two singularities at each corner.

In this paper, we proposed a new algorithm for solving Stokes equations. The proposed algorithm is based on the Uzawa algorithm and finite element method in [28]. We estimate the solvability of the proposed formulation and special orthogonality form for two singular functions. We also give numerical convergence tests to verify our assertion.

\section{Singular Functions of Stokes Equations}

Let the computational domain $\Omega$ be an open and bounded concave polygon in $\mathbb{R}^{2}$ especially having one reentrant corner. The steady-state Stokes equation is

$$
\begin{array}{rlrl}
-\mu \triangle \mathbf{u}+\nabla p=\mathbf{f}, & & \text { in } \Omega, \\
\nabla \cdot \mathbf{u}=0, & & \text { in } \Omega, \\
\mathbf{u}=0, & \text { on } \partial \Omega,
\end{array}
$$

where $\mathbf{f}$ is a given external force field causing an acceleration of the flow in $\mathbf{H}^{-1}(\Omega), \Omega$ is a computational domain in $\mathbb{R}^{2}$, and $\mu=\mathrm{Re}^{-1}$ is the reciprocal of the Reynolds number. The unknowns are the (vector) velocity field $\mathbf{u} \in \mathbf{H}_{0}^{1}(\Omega)$ and the (scalar) pressure $p \in L_{0}^{2}(\Omega)$. The pressure gradient plays a role in an additional force, which prevents a change in the density. In particular, high pressure builds up at points, where, otherwise, a source of the sink would be created. Mathematically, the pressure can be considered as a Lagrange multiplier. Besides, the weak formulation of the Stokes equations leads to a saddle point problem with the restriction $\nabla \cdot \mathbf{u}=0$.

There are mainly two approaches to find finite element approximation of solutions of (3). One approach is called the mixed finite element method which solves velocities and pressure simultaneously by constructing a big linear system. Another approach is an iteration method called the Uzawa method which solves velocity variables involving augmented Lagrangian operator and then updates pressure variable by Richardson update. The advantage of the Uzawa method is that it uses less memory because it solves velocity and pressure separately. However, the iteration process sometimes takes more computational time. The Uzawa iteration can be extended to projection-type methods such as the gaugeUzawa method to solve unsteady incompressible Navier-Stokes equations.

If the solution of (3) is smooth enough, namely, $(\mathbf{u}, p) \in \mathbf{H}^{s+1}(\Omega) \times H^{s}(\Omega)$ with $s \geq 1$, and if a suitable finite element pair is imposed for velocity and pressure, then the finite element solution $\left(\mathbf{u}_{h}, p_{h}\right)$ using the standard mixed finite element method has optimal error bounds as shown in [14]:

$$
\left\|\mathbf{u}-\mathbf{u}_{h}\right\|_{0}+h\left\|\mathbf{u}-\mathbf{u}_{h}\right\|_{1}+h\left\|p-p_{h}\right\|_{0} \leq C h^{s+1}\left(\|\mathbf{u}\|_{s+1}+\|p\|_{s}\right),
$$

where $h$ is the biggest mesh size. However, if $s<1$, then the error bounds only become

$$
\left\|\mathbf{u}-\mathbf{u}_{h}\right\|_{0}+h^{s}\left\|\mathbf{u}-\mathbf{u}_{h}\right\|_{1}+h^{s}\left\|p-p_{h}\right\|_{0} \leq C h^{2 s}\left(\|\mathbf{u}\|_{s+1}+\|p\|_{s}\right) \text {. }
$$

For the case $s<1$, we call the solution $(\mathbf{u}, p)$ a singular solution, otherwise a regular solution. Since singularities are due to re-entrant corners of a computational domain $\Omega$, we assume that $\Omega$ is open and bounded polygonal domain in $\mathbb{R}^{2}$ with one re-entrant corner.

To derive singular and dual singular functions for the Stokes equations, the polar coordinate $(r, \theta)$ of homogeneous Stokes system (3) should be considered, and we can find the singular and dual singular solution via solving the homogeneous system with the separation of variables. Then, we arrive at the singular function of system (3): 


$$
\left(\begin{array}{c}
u^{s} \\
v^{s} \\
p^{s}
\end{array}\right)=C_{1}\left(\begin{array}{c}
\frac{r^{\lambda}}{\mu} \lambda \sin (\theta) \sin ((1-\lambda) \theta) \\
\frac{r^{\lambda}}{\mu}(\sin (\lambda \theta)-\lambda \sin (\theta) \cos ((1-\lambda) \theta)) \\
-2 r^{\lambda-1} \lambda \cos ((1-\lambda) \theta)
\end{array}\right.
$$

where $\lambda$ is solution of

$$
\lambda^{2} \sin ^{2}(\omega)=\sin ^{2}(\lambda \omega)
$$

and $C_{1}$ and $C_{2}$ are

$$
-C_{2}\left(\begin{array}{c}
\frac{r^{\lambda}}{\mu}(\sin (\lambda \theta)+\lambda \sin (\theta) \cos ((1-\lambda) \theta)) \\
\frac{r^{\lambda}}{\mu} \lambda \sin (\theta) \sin ((1-\lambda) \theta) \\
2 r^{\lambda-1} \lambda \sin ((1-\lambda) \theta)
\end{array}\right)
$$

$$
\begin{aligned}
& C_{1}=\sin (\lambda \omega)+\lambda \sin (\omega) \cos ((1-\lambda) \omega), \\
& C_{2}=\lambda \sin (\omega) \sin ((1-\lambda) \omega) .
\end{aligned}
$$

Similarly, the dual singular functions are derived by

$$
\left(\begin{array}{c}
u^{d} \\
v^{d} \\
p^{d}
\end{array}\right)=D_{1}\left(\begin{array}{c}
-\frac{r^{-\lambda}}{\mu} \lambda \sin (\theta) \sin ((1+\lambda) \theta) \\
-\frac{r^{-\lambda}}{\mu}(\sin (\lambda \theta)-\lambda \sin (\theta) \cos ((1+\lambda) \theta) \\
2 r^{-\lambda-1} \lambda \cos ((1+\lambda) \theta)
\end{array}\right.
$$$$
+D_{2}\left(\begin{array}{c}
\frac{r^{-\lambda}}{\mu}(\sin (\lambda \theta)+\lambda \sin (\theta) \cos ((1+\lambda) \theta)) \\
\frac{r^{-\lambda}}{\mu} \lambda \sin (\theta) \sin ((1+\lambda) \theta) \\
2 r^{-\lambda-1} \lambda \sin ((1+\lambda) \theta)
\end{array}\right)
$$

where $\lambda$ is solution of (7) and $D_{1}$ and $D_{2}$ are

$$
\begin{aligned}
& D_{1}=\sin (\lambda \omega)+\lambda \sin (\omega) \cos ((1+\lambda) \omega), \\
& D_{2}=\lambda \sin (\omega) \sin ((1+\lambda) \omega) .
\end{aligned}
$$

The following lemma describes the number of singular functions depending on the value of $\omega$ and the relationship between values of $\lambda$ and $\omega$ is shown in Figure 1.

Lemma 1 (see [26]). For $\chi: \approx 1.430296653124203$, we have the following properties:

(1) $\lambda=0$ and $\lambda=1$ are solutions for any $\omega$. We call these trivial solutions.

(2) There are only trivial solutions for $0 \leq \omega \leq \pi$. It means that there is no $(\mathbf{u}, p) \notin \mathbf{H}^{2}(\Omega) \times H^{1}(\Omega)$, solution of Stokes equation with homogeneous boundary condition on $\Gamma_{\text {in }}$, where $\Gamma_{\text {in }}$ is the boundary near re-entrant corner.

(3) If $\pi \leq \omega \leq \chi \pi$, then there is a unique solution $\lambda$ except trivial solutions. Therefore, there is one singular solution $(\mathbf{u}, p) \in \mathbf{H}^{1}(\Omega) \times L^{2}(\Omega)$.

(4) If $\chi \pi<\omega<2 \pi$, then there are two solutions $\lambda$ except trivial solutions. Thus, there is two singular solutions $(\mathbf{u}, p) \in \mathbf{H}^{1}(\Omega) \times L^{2}(\Omega)$.

(5) If $\omega=2 \pi$, then solution is $\lambda=0.5$ except trivial solutions.

It is shown that 2 singular functions of solution (3) can be involved in each reentrant corner. If there is one reentrant corner, the solution of (3) can be written by the form

$$
\left(\begin{array}{l}
\mathbf{u} \\
p
\end{array}\right)=\left(\begin{array}{l}
\mathbf{w} \\
q
\end{array}\right)+\alpha_{1}\left(\begin{array}{l}
\mathbf{u}_{1}^{s} \\
p_{1}^{s}
\end{array}\right)+\alpha_{2}\left(\begin{array}{c}
\mathbf{u}_{2}^{s} \\
p_{2}^{s}
\end{array}\right)
$$

where $\alpha_{1}$ and $\alpha_{2}$ are the stress intensity factors, $\left(\mathbf{u}_{i}^{s}, p_{i}^{s}\right) \notin \mathbf{H}^{2}(\Omega) \times H^{1}(\Omega), i=1,2$, are singular functions of (6), and $(\mathbf{w}, q) \in \mathbf{H}^{2}(\Omega) \times H^{1}(\Omega)$ is the regular solution.

Lemma 2 (see [26]). The singular function $\left(\mathbf{u}_{i}^{s}, p_{i}^{s}\right) \in \mathbf{H}^{1+\lambda}$ $(\Omega) \times H^{\lambda}(\Omega)$ and the dual singular function $\left(\mathbf{u}_{i}^{d}, p_{i}^{d}\right) \notin \mathbf{H}^{1}$ $(\Omega) \times L^{2}(\Omega), i=1,2$, satisfy

$$
\begin{aligned}
-\mu \Delta \mathbf{u}_{i}^{s}+\nabla p_{i}^{s}=0, & \text { in } \Omega, \\
\nabla \cdot \mathbf{u}_{i}^{s}=0, & \text { on } \Gamma_{\mathrm{in}}, \\
-\mu \Delta \mathbf{u}_{i}^{d}+\nabla p_{i}^{d}=0, & \text { in } \Omega, \\
\nabla \cdot \mathbf{u}_{i}^{d}=0, & \text { on } \Gamma_{\mathrm{in}},
\end{aligned}
$$

respectively. The boundary conditions of $\mathbf{u}_{i}^{s}$ and $\mathbf{u}_{i}^{d}$ vanish on $\Gamma_{i n}$, but the boundary value of $\mathbf{u}_{i}^{d}$ is not defined at the origin. Both of $\mathbf{u}_{i}^{s}$ and $\mathbf{u}_{i}^{d}$ are not $\mathbf{0}$ on $\Gamma_{\text {out }}=\partial \Omega / \Gamma_{\text {in }}$.

\section{FE-DSFM and Uzawa Iteration}

Uzawa method solves velocity variables involving augmented Lagrangian operator and then updates the pressure variable by Richardson update. Algorithm 1 is the Uzawa method for solving stokes equation (3).

There are some theoretical works of literature on the Uzawa method and augmented Lagrangian method. In [29-32], the convergence of Algorithm 1 is proved. Especially, it is shown that $\kappa=0$ and $\beta=1$ have optimal 


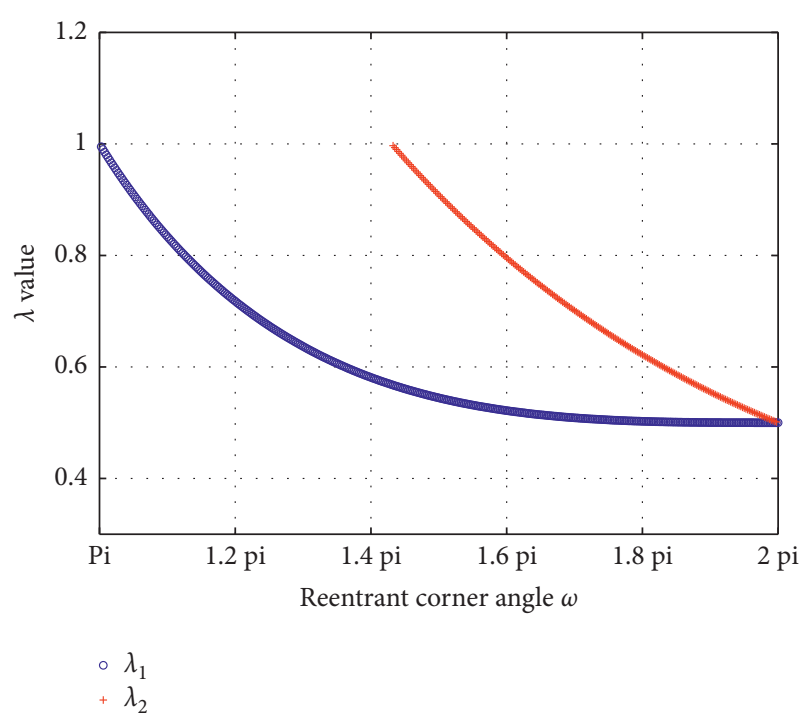

FIGURE $1: \lambda_{1}$ and $\lambda_{2}$ values by numerical calculation by the bisection root-finding method.

Step 1: find $\mathbf{u}^{n+1} \in \mathbf{H}_{0}^{1}(\omega)$ as the solution of $-\mu \Delta \mathbf{u}^{n+1}-\kappa \nabla\left(\nabla \cdot \mathbf{u}^{n+1}\right)+\nabla p^{n}=\mathbf{f}$, in $\Omega$

Step 2: update $p^{n+1} \in L^{2}(\Omega)$ from the Richardson update $p^{n+1}=p^{n}-\beta \mu \nabla \cdot \mathbf{u}^{n+1}$

Algorithm 1: (Uzawa method). Given any initial guess $p^{0} \in L^{2}(\Omega)$, repeat the following steps until $\left\|\mathbf{u}^{n+1}-\mathbf{u}^{n}\right\| \leq$ tolerance:

convergence in [31]. So, we choose $\kappa=0$ and $\beta=1$ for our algorithms.

Our goal is to construct FE-DSFM with the Uzawa iteration. We consider Stokes equations with polygonal domain including one reentrant corner. To represent FEDSFM, we first introduce the cut-off function. Let $\omega$ be the internal angle of $\Omega$ satisfying $\pi<\omega<2 \pi$. Now, we set

$$
B\left(r_{1} ; r_{2}\right)=\left\{(r, \theta): r_{1}<r<r_{2} \text { and } 0<\theta<\omega\right\} \cap \Omega,
$$

and $B\left(r_{1}\right)=B\left(0 ; r_{1}\right)$ in the polar coordinates $(r, \theta)$ and define cut-off function

$$
\eta_{\rho}(r)= \begin{cases}1, & \text { in } B\left(\frac{1}{2} \rho R\right), \\ \delta(r), & \text { in } B\left(\frac{1}{2} \rho R ; \rho R\right), \\ 0, & \text { in } \Omega \backslash B(\rho R),\end{cases}
$$

where $\delta(r)$ is very smooth function, $\rho$ is a parameter in $(0,2]$, and $R$ is a fixed real number which will be determined later so that $\eta_{2 \rho}$ has 0 on whole $\Gamma_{\text {out }}$.
We note that the singular function $(6)(i=1,2)$ are the solutions of homogeneous Stokes equation (3) with vanishing Dirichlet boundary condition on $\Gamma_{\text {in }}$. Also, $\lambda_{i}$ has to be a positive real number and $\left(\mathbf{u}_{i}^{s}, p_{i}^{s}\right) \in \mathbf{H}^{1+\lambda_{i}}(\Omega) \times H^{\lambda_{i}}(\Omega), i=1,2$. Let $\eta$ be a smooth cut-off function (14) with smooth function which is equal to the one identically in the neighborhood of origin, and the support of $\eta$ is small enough so that the functions $\eta \mathbf{u}_{i}^{s}$, $i=1,2$, vanish identically on $\partial \Omega$. Then, in general, the solution $(\mathbf{u}, p)$ including singular parts of (3) can be rewritten in the form (11):

$$
\left(\begin{array}{l}
\mathbf{u} \\
p
\end{array}\right)=\left(\begin{array}{l}
\mathbf{w} \\
q
\end{array}\right)+\alpha_{1}\left(\begin{array}{c}
\eta_{1} \mathbf{u}_{1}^{s} \\
\eta_{1} p_{1}^{s}
\end{array}\right)+\alpha_{2}\left(\begin{array}{c}
\eta_{2} \mathbf{u}_{2}^{s} \\
\eta_{2} p_{2}^{s}
\end{array}\right),
$$

where $\alpha_{1}$ and $\alpha_{2}$ are the stress intensity factors and $(\mathbf{w}, q) \in \mathbf{H}^{2}(\Omega) \times H^{1}(\Omega)$.

The strategy of FE-DSFM is to find the regular solution $(\mathbf{w}, q) \in \mathbf{H}^{2}(\Omega) \times H^{1}(\Omega)$ and stress intensity factors $\alpha_{1}$ and $\alpha_{2}$ by applying the standard Uzawa method with an additional equation. To make simpler formula, we assume that there is only one singular solution, that is, one of $\alpha_{1}$ or $\alpha_{2}$ is zero. Let $\mathbf{u}^{n+1}=\mathbf{w}^{n+1}+\alpha^{n+1} \eta_{\rho} \mathbf{u}^{s}$ and $p^{n}=q^{n}+\alpha^{n} \eta_{\rho} p^{s}$. Then, Step 1 of the Uzawa algorithm can be

$$
-\mu \Delta\left(\mathbf{w}^{n+1}+\alpha^{n+1} \eta_{\rho} \mathbf{u}^{s}\right)+\nabla\left(q^{n}+\alpha^{n} \eta_{\rho} p^{s}\right)=\mathbf{f}
$$

Even though $-\mu \Delta \eta_{\rho} \mathbf{u}^{s}$ and $\nabla \eta_{\rho} p^{s} \notin \mathbf{L}^{2}$, we know that $-\mu \triangle\left(\eta_{\rho} \mathbf{u}^{s}\right)+\nabla\left(\eta_{\rho} p^{s}\right) \in \mathbf{L}^{2}$. So, (16) can be rewritten as

$$
-\mu \Delta \mathbf{w}^{n+1}+\nabla q^{n}+\alpha^{n+1}\left(-\mu \Delta \eta_{\rho} \mathbf{u}^{s}+\nabla \eta_{\rho} p^{s}\right)=\mathbf{f} .
$$

For the sake of a clear explanation, we note that the inner product of vectors $\mathbf{a}=\left(a_{1}, a_{2}\right)$ and $\mathbf{b}=\left(b_{1}, b_{2}\right)$ is

$$
\begin{aligned}
\langle\mathbf{a}, \mathbf{b}\rangle= & \left\langle a_{1}, b_{1}\right\rangle+\left\langle a_{2}, b_{2}\right\rangle, \\
\langle\nabla \mathbf{a}, \nabla \mathbf{b}\rangle= & \left\langle\partial_{x} a_{1}, \partial_{x} b_{1}\right\rangle+\left\langle\partial_{x} a_{2}, \partial_{x} b_{2}\right\rangle \\
& +\left\langle\partial_{y} a_{1}, \partial_{y} b_{1}\right\rangle+\left\langle\partial_{y} a_{2}, \partial_{y} b_{2}\right\rangle .
\end{aligned}
$$

Then, the weak formulation of (17) is

$$
\begin{aligned}
& \mu\left\langle\nabla \mathbf{w}^{n+1}, \nabla \mathbf{v}\right\rangle+\left\langle\nabla q^{n}, \mathbf{v}\right\rangle+\alpha^{n+1}\left\langle-\mu \Delta \eta_{\rho} \mathbf{u}^{s}+\nabla \eta_{\rho} p^{s}, \mathbf{v}\right\rangle \\
& \quad=\langle\mathbf{f}, \mathbf{v}\rangle,
\end{aligned}
$$

for all $\mathbf{v} \in \mathbf{H}_{0}^{1}(\Omega)$. Since $q^{n} \in H^{1}(\Omega)$ and $-\mu \Delta \eta_{\rho} \mathbf{u}^{s}+\nabla \eta_{\rho}$ $p^{s} \in \mathbf{L}^{2}(\Omega)$, equation (19) is solvable, and it has unique solution $\mathbf{w}^{n+1} \in \mathbf{H}_{0}^{1}(\Omega)$ if we know $\alpha^{n+1} \in \mathbb{R}$. However, since $\alpha^{n+1}$ is unknown, we need one more equation, and it should be

(i) A single equation because $\alpha$ is a real number

(ii) Linearly independent to (19)

(iii) $\alpha$ which is not disappeared

(iv) Solvable near the singularity corner

Since equation (19) is a Poisson-type problem to find $\mathbf{w}^{n+1}$, we can make the additional equation by testing the dual singular function of the following Poisson equation, instead of that of Stokes equation (9): 


$$
s_{L}^{d}(r, \theta)=r^{-(\pi / \omega)} \sin \left(\frac{\pi}{\omega} \theta\right)
$$

Lemma 3 (see [19]). The function $s_{L}^{d}$ in (20) has the following properties:

(1) $s_{L}^{d}$ is not defined at origin

(2) $s_{L}^{d} \in L^{2}$ and $s_{L}^{d} \notin H^{1}$

(3) $-\triangle s_{L}^{d}=0$

(4) $s_{L}^{d}=0$ on $=\Gamma_{\text {in }}$ (except origin)
(5) For all $u \in H_{0}^{1} \cap H^{2},\left\langle-\Delta u, \eta_{2 \rho} s_{L}^{d}\right\rangle=\left\langle u,-\Delta \eta_{2 \rho} s_{L}^{d}\right\rangle$

We denote the vector form of (20), $\eta_{2 \rho} \mathbf{S}_{L}^{d}=\left(\begin{array}{l}\eta_{2 \rho} s_{L} \\ \eta_{2 \rho} s_{L}\end{array}\right)$. We now test $\eta_{2 \rho} \mathbf{S}_{L}^{d}$ to (17) and apply Lemma 3 to obtain

$$
\begin{aligned}
& \mu\left\langle\mathbf{w}^{n+1},-\Delta \eta_{2 \rho} \mathbf{S}_{L}^{d}\right\rangle+\left\langle\nabla q^{n}, \eta_{2 \rho} \mathbf{S}_{L}^{d}\right\rangle \\
& \quad+\alpha^{n+1}\left\langle-\mu \Delta \eta_{\rho} \mathbf{u}^{s}+\nabla \eta_{\rho} p^{s}, \eta_{2 \rho} \mathbf{S}_{L}^{d}\right\rangle=\left\langle\mathbf{f}, \eta_{2 \rho} \mathbf{S}_{L}^{d}\right\rangle .
\end{aligned}
$$

Let $\beta_{s}=-\Delta \eta_{\rho} \mathbf{u}^{s}+\left\langle\nabla \eta_{\rho} p^{s}, \eta_{2 \rho} \mathbf{S}_{L}^{d}\right\rangle$ and $\beta_{f}=\left\langle\mathbf{f}, \eta_{2 \rho} \mathbf{S}_{L}^{d}\right\rangle$ $-\left\langle\nabla q^{n}, \eta_{2 \rho} \mathbf{S}_{L}^{d}\right\rangle$. Then, we have the system

$$
\left\{\begin{array}{l}
\mu\left\langle\nabla \mathbf{w}^{n+1}, \nabla \mathbf{v}\right\rangle+\alpha^{n+1}\left\langle-\mu \Delta \eta_{\rho} \mathbf{u}^{s}+\nabla \eta_{\rho} p^{s}, \mathbf{v}\right\rangle=\langle\mathbf{f}, \mathbf{v}\rangle-\left\langle\nabla q^{n}, \mathbf{v}\right\rangle, \\
\alpha^{n+1}=\frac{\beta_{f}}{\beta_{s}}-\frac{1}{\beta_{s}}\left\langle\mathbf{w}^{n+1},-\Delta \eta_{2 \rho} \mathbf{S}_{L}^{d}\right\rangle .
\end{array}\right.
$$

We first check solvability of system (22).

Lemma 4. Define a mapping $T: \mathbb{R} \longrightarrow \mathbf{H}^{1}$ and $F: \mathbf{H}^{1} \longrightarrow \mathbb{R}$ such that $T(\alpha)=w$, where

$$
\left\{\begin{array}{l}
\mu\langle\nabla \mathbf{w}, \nabla \mathbf{v}\rangle+\alpha\left\langle-\mu \Delta \eta_{\rho} \mathbf{u}^{s}+\nabla \eta_{\rho} p^{s}, \mathbf{v}\right\rangle=\langle\mathbf{f}, \mathbf{v}\rangle-\langle\nabla q, \mathbf{v}\rangle, \\
F(\mathbf{w})=\frac{\beta_{f}}{\beta_{s}}-\frac{1}{\beta_{s}}\left\langle\mathbf{w},-\Delta \eta_{2 \rho} \mathbf{S}_{L}^{d}\right\rangle .
\end{array}\right.
$$

Given a function $f, \nabla q^{n} \in \mathbf{L}^{2}$,

$$
\|(F \circ T)\|=0 \text {. }
$$

Therefore, there is a unique value $\gamma$ such that $(F \circ T)\left(\gamma^{*}\right)=\gamma$ for all $\gamma^{*} \in \mathbb{R}$.

Proof. Let $\alpha_{1}, \alpha_{2} \in \mathbb{R}$ with $\alpha_{1} \neq \alpha_{2}, \quad T\left(\alpha_{1}\right)=\mathbf{w}_{1}$, and $T\left(\alpha_{2}\right)=\mathbf{w}_{2}$. Define two equations:

$$
\begin{aligned}
\mu\langle\nabla \mathbf{a}, \nabla \mathbf{v}\rangle+\left\langle\nabla q^{n}, \mathbf{v}\right\rangle & =\langle\mathbf{f}, \mathbf{v}\rangle, \\
\langle\mu \nabla \mathbf{z}, \nabla \mathbf{v}\rangle+\left\langle-\mu \Delta \eta_{\rho} \mathbf{u}^{s}+\nabla \eta_{\rho} p^{s}, \mathbf{v}\right\rangle & =0 .
\end{aligned}
$$
Then,

Then, $T\left(\alpha_{1}\right)=\mathbf{w}_{1}=\mathbf{a}+\alpha_{1} \mathbf{z}$ and $T\left(\alpha_{2}\right)=\mathbf{w}_{1}=\mathbf{a}+\alpha_{2} \mathbf{z}$.

$$
\begin{aligned}
\left|(F \circ T)\left(\alpha_{2}\right)-(F \circ T)\left(\alpha_{1}\right)\right| & =\left|\frac{1}{\beta_{s}}\left\langle\mathbf{w}_{1}-\mathbf{w}_{2},-\Delta \eta_{2 \rho} \mathbf{S}_{L}^{d}\right\rangle\right| \\
& =\left|\frac{1}{\beta_{s}}\left\langle\left(\alpha_{1}-\alpha_{2}\right) \mathbf{z},-\Delta \eta_{2 \rho} \mathbf{S}_{L}^{d}\right\rangle\right| \\
& =\left|\frac{\left(\alpha_{1}-\alpha_{2}\right)}{\beta_{s}}\left\langle\mathbf{z},-\Delta \eta_{2 \rho} \mathbf{S}_{L}^{d}\right\rangle\right| .
\end{aligned}
$$

We note that $-\mu \Delta \eta_{\rho} \mathbf{u}^{s}+\nabla \eta_{\rho} p^{s}=0$ in $\Omega \backslash B(\rho R)$ and so is $\mathbf{z}$ with $\mathbf{z}=0$ on $\partial \Omega$. Since $-\Delta \eta_{2 \rho} \mathbf{S}_{L}^{d}=0$ in $B(\rho R)$, we have

$$
\left\langle\mathbf{z},-\Delta \eta_{2 \rho} \mathbf{S}_{L}^{d}\right\rangle=0 .
$$

Theorem 1. Formulation (22) has a unique solution $\mathbf{w}$ in $\mathbf{H}^{2}(\Omega) \cap \mathbf{H}_{0}^{1}(\Omega)$.

Proof. By Lemma 4, contract mapping theorem provides the existence of the unique fixed point of $F \circ T$.

We now define finite element space similarly as in previous sections to construct fully discrete FE-DSFM. Let $\mathfrak{I}=\{K\}$ be a shape-regular quasi-uniform partition of $\Omega$ of mesh size $h$ into closed elements $K$. The vector and scalar finite element spaces are

$$
\begin{aligned}
\mathbb{W}_{h} & :=\left\{\mathbf{w}_{h} \in \mathbf{L}^{2}(\Omega):\left.\mathbf{w}_{h}\right|_{K} \in \mathscr{P}(K), \forall K \in \mathfrak{I}\right\}, \\
\mathbb{V}_{h} & :=\mathbb{W}_{h} \cap \mathbf{H}_{0}^{1}(\Omega), \\
\mathbb{P}_{h} & :=\left\{q_{h} \in L^{2}(\Omega) \cap C^{0}(\Omega):\left.q_{h}\right|_{K} \in \mathbb{Q}(K), \forall K \in \mathfrak{I}\right\},
\end{aligned}
$$


where $\mathscr{P}(K)$ and $\mathscr{Q}(K)$ are spaces of polynomials with degree bounded uniformly with respect to $K \in \mathfrak{T}$. We stress that the space $\mathbb{P}_{h}$ is composed of continuous functions to use integration by parts:

$$
\left\langle\nabla \cdot \mathbf{v}_{h}, q_{h}\right\rangle=-\left\langle\mathbf{v}_{h}, \nabla q_{h}\right\rangle, \quad \forall \mathbf{v}_{h} \in \mathbb{V}_{h},
$$

for all $q_{h} \in \mathbb{P}_{h}$. Then, the finite element approximation for (22) is to find $\mathbf{w}_{h} \in \mathbb{V}_{h}$ and $\alpha_{h}^{n+1} \in \mathbb{R}$ such that

$$
\left\{\begin{array}{l}
\mu\left\langle\nabla \mathbf{w}_{h}^{n+1}, \nabla \mathbf{v}_{h}\right\rangle+\alpha_{h}^{n+1}\left\langle-\mu \Delta \eta_{\rho} \mathbf{u}^{s}+\nabla \eta_{\rho} p^{s}, \mathbf{v}_{h}\right\rangle=\left\langle\mathbf{f}, \mathbf{v}_{h}\right\rangle-\left\langle\nabla q_{h}^{n}, \mathbf{v}_{h}\right\rangle \\
\alpha_{h}^{n+1}=\frac{\beta_{f h}}{\beta_{s}}-\frac{1}{\beta_{s}}\left\langle\mathbf{w}_{h}^{n+1},-\Delta \eta_{2 \rho} \mathbf{S}_{L}^{d}\right\rangle
\end{array}\right.
$$

where $\beta_{f h}=\left\langle\mathbf{f}, \eta_{2 \rho} \boldsymbol{S}_{L}^{d}\right\rangle-\left\langle\nabla q_{h}^{n}, \eta_{2 \rho} \mathbf{S}_{L}^{d}\right\rangle$. And, the matrix form of the coupled system (30) becomes

$$
\left(A+a \cdot b^{T}\right) \mathbf{w}_{h}=f,
$$

where $A$ is symmetric positive definite square matrix and $a$ and $b$ are column vectors. System (31) can be solved by the Sherman-Morrison formula:

$$
\left(A+a \cdot b^{T}\right)^{-1}=A^{-1}-\frac{A^{-1} a b^{T} A^{-1}}{1+b^{T} A^{-1} a},
$$

and $\alpha_{h}^{n+1}$ can be computed by second equation of (30).

Sherman-Morrison formula is a rigorous approach to solve system (30), but it is sometimes complicated to apply for some problems including many singular functions. Since $(F \circ T)\left(\alpha^{n}\right)=\alpha^{n+1}$ by Lemma 4 , we choose $\alpha_{h}^{n}$ instead of $\alpha_{h}^{n+1}$ in first equation of (30). Algorithm 2 is the proposed method for one singular function.

Remark 1. Compared with the original Uzawa iteration (Algorithm 1), the proposed method (Algorithm 2) needs only one more linear solver for the initial process.

\section{Algorithm for Two Singular Functions}

In this section, we construct an algorithm for two singularities in one corner. We consider the solution is

$$
\left(\begin{array}{l}
\mathbf{u} \\
p
\end{array}\right)=\left(\begin{array}{l}
\mathbf{w} \\
q
\end{array}\right)+\alpha_{1}\left(\begin{array}{l}
\eta_{\rho} \mathbf{u}_{1}^{s} \\
\eta_{\rho} p_{1}^{s}
\end{array}\right)+\alpha_{2}\left(\begin{array}{c}
\eta_{\rho} \mathbf{u}_{2}^{s} \\
\eta_{\rho} p_{2}^{s}
\end{array}\right) .
$$

With the implicit formation of two stress intensity factors, step 1 of Uzawa algorithm is

$$
\begin{gathered}
-\mu \Delta \mathbf{w}^{n+1}+\nabla q^{n}+\alpha_{1}^{n+1}\left(-\Delta \eta_{\rho} \mathbf{u}_{1}^{s}+\nabla \eta_{\rho} p_{1}^{s}\right) \\
+\alpha_{2}^{n+1}\left(-\Delta \eta_{\rho} \mathbf{u}_{2}^{s}+\nabla \eta_{\rho} p_{2}^{s}\right)=\mathbf{f} .
\end{gathered}
$$

Here, the unknowns are $\mathbf{w}^{n+1}, \alpha_{1}^{n+1}$, and $\alpha_{2}^{n+1}$. However, our test function which is not in $\mathbf{H}_{0}^{1}$ is only $\eta_{2 \rho} \mathbf{S}_{L}^{d}$. Instead of finding another test function, we use the following property.

Theorem 2. (properties of singular functions). Let $\mathbf{u}_{1}^{s}=$ $\left(\begin{array}{c}u_{1}^{s} \\ v_{1}^{s}\end{array}\right)$ and $\mathbf{u}_{2}^{s}=\left(\begin{array}{c}u_{2}^{s} \\ v_{2}^{s}\end{array}\right)$ be the singular functions of Stokes equation on same corner. Then,

$$
\begin{aligned}
\langle & \left.-\Delta \eta_{\rho} u_{1}^{s}+\left(\eta_{\rho} p_{1}^{s}\right)_{x}, \eta_{2 \rho} s_{L}^{d}\right\rangle\left\langle-\Delta \eta_{\rho} u_{2}^{s}+\left(\eta_{\rho} p_{2}^{s}\right)_{x}, \eta_{2 \rho} s_{L}^{d}\right\rangle \\
& +\left\langle-\Delta \eta_{\rho} v_{1}^{s}+\left(\eta_{\rho} p_{1}^{s}\right)_{y}, \eta_{2 \rho} s_{L}^{d}\right\rangle\left\langle-\Delta \eta_{\rho} v_{2}^{s}+\left(\eta_{\rho} p_{2}^{s}\right)_{y}, \eta_{2 \rho} s_{L}^{d}\right\rangle=0 .
\end{aligned}
$$

Proof. To tell the conclusion, it is too difficult to show equation (35) by algebraic calculation because the integral functions depend on some constants which are constructed by solutions of transcendental function.

First of all, from singular function (6) and cut-off function (14) with smooth function, we obtain

$$
\begin{aligned}
& -\Delta \eta_{\rho} \mathbf{u}_{s}+\nabla \eta_{\rho} p_{s} \\
& =-C_{1}\left(\frac{\partial^{2} \eta_{\rho}}{\partial r^{2}} r^{\lambda}+(2 \lambda+1) \frac{\partial \eta_{\rho}}{\partial r} r^{\lambda-1}\right)\left(\begin{array}{c}
\lambda \sin (\theta) \sin ((1-\lambda) \theta) \\
\sin (\lambda \theta)-\lambda \sin (\theta) \cos ((1-\lambda) \theta)
\end{array}\right) \\
& -C_{1}\left(2 \lambda \frac{\partial \eta_{\rho}}{\partial r} r^{\lambda-1}\right)\left(\begin{array}{c}
\cos (\theta) \cos ((1-\lambda) \theta) \\
\sin (\theta) \cos ((1-\lambda) \theta)
\end{array}\right)
\end{aligned}
$$


Step 1: for all $\mathbf{v}_{h} \in \mathbb{W}_{h}$, find $\widehat{w}_{h}^{n+1} \in \mathbb{W}_{h}$ as the solution of $\left\langle\mu \nabla \widehat{w}_{h}^{n+1}, \nabla \mathbf{v}_{h}\right\rangle+\left\langle\nabla q_{h}^{n}, \nabla \mathbf{v}_{h}\right\rangle+\alpha_{h}^{n}\left\langle-\Delta \eta_{\rho} \mathbf{u}^{s}+\nabla \eta_{\rho} p^{s}, \mathbf{v}_{h}\right\rangle=\left\langle\mathbf{f}, \mathbf{v}_{h}\right\rangle$

Step 2: find $\alpha_{h}^{n+1} \in \mathbb{R}$ from the equality $\alpha_{h}^{n+1}=\left(\beta_{f h} / \beta_{s}\right)-\left(1 / \beta_{s}\right)\left\langle\widehat{w}_{h}^{n+1},-\Delta \eta_{2 \rho} \mathbf{S}_{L}^{d}\right\rangle$

Step 3: update $\mathbf{w}_{h}^{n+1} \in \mathbb{W}_{h}$ by $\mathbf{w}_{h}^{n+1}=\widehat{w}_{h}^{n+1}+\left(\alpha_{h}^{n+1}-\alpha_{h}^{n}\right) \mathbf{z}$

Step 4: for all $p_{h} \in \mathbb{P}_{h}$, update $q_{h}^{n+1}$ by $\left\langle q_{h}^{n+1}, p_{h}\right\rangle=\left\langle q_{h}^{n}, p_{h}\right\rangle-\mu\left\langle\nabla \cdot \mathbf{w}_{h}^{n+1}, p_{h}\right\rangle-\alpha_{h}^{n+1}\left\langle\nabla \cdot \eta_{\rho} \mathbf{u}^{s}, p_{h}\right\rangle$

Algorithm 2: (FE-DSFM Uzawa iteration for one singular function). Start with any initial values $p_{h}^{0}, \alpha_{h}^{0}$, and $\mathbf{z}_{h}$ as the solution of $\mu\left\langle\nabla \mathbf{z}_{h}, \nabla \mathbf{v}_{h}\right\rangle+\left\langle-\mu \Delta \eta_{\rho} \mathbf{u}^{s}+\nabla \eta_{\rho} p^{s}, \mathbf{v}_{h}\right\rangle=0$, for all $\mathbf{v}_{h} \in \mathbb{W}_{h}$. Repeat the following steps until $\left\|\mathbf{w}_{h}^{n+1}-\mathbf{w}_{h}^{n}\right\| \leq$ tolerance:

$$
\begin{aligned}
& +C_{2}\left(\frac{\partial^{2} \eta_{\rho}}{\partial r^{2}} r^{\lambda}+(2 \lambda+1) \frac{\partial \eta_{\rho}}{\partial r} r^{\lambda-1}\right)\left(\begin{array}{c}
\sin (\lambda \theta)+\lambda \sin (\theta) \cos ((1-\lambda) \theta) \\
\lambda \sin (\theta) \sin ((1-\lambda) \theta)
\end{array}\right) \\
& -C_{2}\left(2 \lambda \frac{\partial \eta_{\rho}}{\partial r} r^{\lambda-1}\right)\left(\begin{array}{c}
\cos (\theta) \sin ((1-\lambda) \theta) \\
\sin (\theta) \sin ((1-\lambda) \theta)
\end{array}\right) .
\end{aligned}
$$

Let

$$
\begin{aligned}
U A_{\lambda}(\theta)= & -C_{1}(\lambda \sin (\theta) \sin ((1-\lambda) \theta))+C_{2}(\sin (\lambda \theta)+\lambda \sin (\theta) \cos ((1-\lambda) \theta)), \\
U B_{\lambda}(\theta)= & -C_{1}((2 \lambda+1) \lambda \sin (\theta) \sin ((1-\lambda) \theta)+2 \lambda \cos (\theta) \cos ((1-\lambda) \theta)) \\
& +C_{2}((2 \lambda+1)(\sin (\lambda \theta)+\lambda \sin (\theta) \cos ((1-\lambda) \theta))-2 \lambda \cos (\theta) \sin ((1-\lambda) \theta)), \\
V A_{\lambda}(\theta)= & -C_{1}(\sin (\lambda \theta)-\lambda \sin (\theta) \cos ((1-\lambda) \theta))+C_{2}(\lambda \sin (\theta) \sin ((1-\lambda) \theta)), \\
V B_{\lambda}(\theta)= & -C_{1}((2 \lambda+1)(\sin (\lambda \theta)-\lambda \sin (\theta) \cos ((1-\lambda) \theta))+2 \lambda \sin (\theta) \cos ((1-\lambda) \theta)) \\
& +C_{2}((2 \lambda+1) \lambda \sin (\theta) \sin ((1-\lambda) \theta)-2 \lambda \sin (\theta) \sin ((1-\lambda) \theta)) .
\end{aligned}
$$

Then,

Since

$$
\begin{aligned}
& -\Delta \eta_{\rho} u_{s}+\left(\nabla \eta_{\rho} p_{s}\right)_{x}=r^{\lambda}\left(\eta_{\rho}\right)_{r r} U A_{\lambda}(\theta)+r^{\lambda-1}\left(\eta_{\rho}\right)_{r} U B_{\lambda}(\theta), \\
& -\Delta \eta_{\rho} v_{s}+\left(\nabla \eta_{\rho} p_{s}\right)_{y}=r^{\lambda}\left(\eta_{\rho}\right)_{r r} V A_{\lambda}(\theta)+r^{\lambda-1}\left(\eta_{\rho}\right)_{r} V B_{\lambda}(\theta) .
\end{aligned}
$$

$$
\begin{aligned}
\langle- & \left.\Delta \eta_{\rho} u_{s}+\left(\nabla \eta_{\rho} p_{s}\right)_{x}, \eta_{2 \rho} s_{L}^{d}\right\rangle \\
& =\iint_{0}^{\omega}\left(-\Delta \eta_{\rho} u_{s}+\left(\nabla \eta_{\rho} p_{s}\right)_{x}\right)\left(\eta_{2 \rho} r^{-(\pi / \omega)}\right) \sin \left(\frac{\pi}{\omega} \theta\right) \mathrm{d} \theta \mathrm{d} r \\
& =\iint_{0}^{\omega}\left(r^{\lambda}\left(\eta_{\rho}\right)_{r r} U A_{\lambda}(\theta)+r^{\lambda-1}\left(\eta_{\rho}\right)_{r} U B_{\lambda}(\theta)\right)\left(\eta_{2 \rho} r^{-(\pi / \omega)}\right) \sin \left(\frac{\pi}{\omega} \theta\right) \mathrm{d} \theta \mathrm{d} r
\end{aligned}
$$




$$
\begin{aligned}
= & \int r^{\lambda}\left(\eta_{\rho}\right)_{r r}\left(\eta_{2 \rho}\right) r^{-(\pi / \omega)} d r \int_{0}^{\omega} U A_{\lambda}(\theta) \sin \left(\frac{\pi}{\omega} \theta\right) \mathrm{d} \theta \\
& +\int r^{\lambda-1}\left(\eta_{\rho}\right)_{r}\left(\eta_{2 \rho}\right) r^{-(\pi / \omega)} d r \int_{0}^{\omega} U B_{\lambda}(\theta) \sin \left(\frac{\pi}{\omega} \theta\right) \mathrm{d} \theta, \\
& \cdot\left\langle-\Delta \eta_{\rho} v_{s}+\left(\nabla \eta_{\rho} p_{s}\right)_{y}, \eta_{2 \rho} s_{L}^{d}\right\rangle \\
= & \int r^{\lambda}\left(\eta_{\rho}\right)_{r r}\left(\eta_{2 \rho}\right) r^{-(\pi / \omega)} \mathrm{d} r \int_{0}^{\omega} V A_{\lambda}(\theta) \sin \left(\frac{\pi}{\omega} \theta\right) \mathrm{d} \theta \\
& +\int r^{\lambda-1}\left(\eta_{\rho}\right)_{r}\left(\eta_{2 \rho}\right) r^{-(\pi / \omega)} \mathrm{d} r \int_{0}^{\omega} V B_{\lambda}(\theta) \sin \left(\frac{\pi}{\omega} \theta\right) \mathrm{d} \theta .
\end{aligned}
$$

We can conclude that equation (35) holds if

$$
\begin{aligned}
& \int_{0}^{\omega} U A_{\lambda 1}(\theta) \sin \left(\frac{\pi}{\omega} \theta\right) \mathrm{d} \theta \int_{0}^{\omega} U A_{\lambda 2}(\theta) \sin \left(\frac{\pi}{\omega} \theta\right) \mathrm{d} \theta \\
& +\int_{0}^{\omega} V A_{\lambda 1}(\theta) \sin \left(\frac{\pi}{\omega} \theta\right) \mathrm{d} \theta \int_{0}^{\omega} V A_{\lambda 2}(\theta) \sin \left(\frac{\pi}{\omega} \theta\right)=0 \\
& \int_{0}^{\omega} U A_{\lambda 1}(\theta) \sin \left(\frac{\pi}{\omega} \theta\right) \mathrm{d} \theta \int_{0}^{\omega} U B_{\lambda 2}(\theta) \sin \left(\frac{\pi}{\omega} \theta\right) \mathrm{d} \theta \\
& +\int_{0}^{\omega} V A_{\lambda 1}(\theta) \sin \left(\frac{\pi}{\omega} \theta\right) \mathrm{d} \theta \int_{0}^{\omega} V B_{\lambda 2}(\theta) \sin \left(\frac{\pi}{\omega} \theta\right)=0 \\
& \int_{0}^{\omega} U B_{\lambda 1}(\theta) \sin \left(\frac{\pi}{\omega} \theta\right) \mathrm{d} \theta \int_{0}^{\omega} U A_{\lambda 2}(\theta) \sin \left(\frac{\pi}{\omega} \theta\right) \mathrm{d} \theta \\
& +\int_{0}^{\omega} V B_{\lambda 1}(\theta) \sin \left(\frac{\pi}{\omega} \theta\right) \mathrm{d} \theta \int_{0}^{\omega} V A_{\lambda 2}(\theta) \sin \left(\frac{\pi}{\omega} \theta\right)=0 \\
& \int_{0}^{\omega} U B_{\lambda 1}(\theta) \sin \left(\frac{\pi}{\omega} \theta\right) \mathrm{d} \theta \int_{0}^{\omega} U B_{\lambda 2}(\theta) \sin \left(\frac{\pi}{\omega} \theta\right) \mathrm{d} \theta \\
& +\int_{0}^{\omega} V B_{\lambda 1}(\theta) \sin \left(\frac{\pi}{\omega} \theta\right) \mathrm{d} \theta \int_{0}^{\omega} V B_{\lambda 2}(\theta) \sin \left(\frac{\pi}{\omega} \theta\right)=0 .
\end{aligned}
$$

However, we could not show equations (40)-(43) are valid by algebraically computation. Therefore, we calculate them numerically.
For numerical substitution, we first compute the above integral forms:

$$
\begin{aligned}
\int_{0}^{\omega} U & A_{\lambda}(\theta) \times \sin \left(\frac{\pi}{\omega} \theta\right) \mathrm{d} \theta \\
= & \frac{1}{4}\left(\frac{\omega}{\lambda \omega-\pi}-\frac{\omega}{\lambda \omega+\pi}\right)\left(C_{1} \lambda(1+\cos (\lambda \omega))-C_{2}(2+\lambda) \sin (\lambda \omega)\right) \\
& +\frac{1}{4}\left(\frac{\omega}{(2-\lambda) \omega+\pi}-\frac{\omega}{(2-\lambda) \omega-\pi}\right) \\
& \times\left(C_{1} \lambda(1+\cos ((2-\lambda) \omega))-C_{2} \lambda \sin ((2-\lambda) \omega)\right)
\end{aligned}
$$




$$
\begin{aligned}
\int_{0}^{\omega} U & B_{\lambda}(\theta) \times \sin \left(\frac{\pi}{\omega} \theta\right) \mathrm{d} \theta \\
= & \frac{1}{4}\left(\frac{\omega}{\lambda \omega-\pi}-\frac{\omega}{\lambda \omega+\pi}\right)\left(C_{1}\left(2 \lambda^{2}+3 \lambda\right)(1+\cos (\lambda \omega))-C_{2}\left(2 \lambda^{2}+7 \lambda+2\right) \sin (\lambda \omega)\right) \\
& +\frac{1}{4}\left(\frac{\omega}{(2-\lambda) \omega+\pi}-\frac{\omega}{(2-\lambda) \omega-\pi}\right) \\
& \times\left(C_{1}\left(2 \lambda^{2}-\lambda\right)(1+\cos ((2-\lambda) \omega))-C_{2}\left(2 \lambda^{2}-\lambda\right) \sin ((2-\lambda) \omega)\right) \\
\int_{0}^{\omega} & V A_{\lambda}(\theta) \times \sin \left(\frac{\pi}{\omega} \theta\right) \mathrm{d} \theta \\
= & \frac{1}{4}\left(\frac{\omega}{\lambda \omega-\pi}-\frac{\omega}{\lambda \omega+\pi}\right)\left(C_{1}(2-\lambda) \sin (\lambda \omega)-\lambda C_{2}(1+\cos (\lambda \omega))\right) \\
& +\frac{1}{4}\left(\frac{\omega}{(2-\lambda) \omega+\pi}-\frac{\omega}{(2-\lambda) \omega-\pi}\right) \\
& \times\left(C_{1} \lambda \sin ((2-\lambda) \omega)+C_{2} \lambda(1+\cos ((2-\lambda) \omega))\right) \\
\int_{0}^{\omega} V & B_{\lambda}(\theta) \times \sin \left(\frac{\pi}{\omega} \theta\right) \mathrm{d} \theta \\
= & \frac{1}{4}\left(\frac{\omega}{\lambda \omega-\pi}-\frac{\omega}{\lambda \omega+\pi}\right)\left(C_{2}\left(\lambda-2 \lambda^{2}\right)(1+\cos (\lambda \omega))-C_{1}\left(2 \lambda^{2}-5 \lambda-2\right) \sin (\lambda \omega)\right) \\
+ & \frac{1}{4}\left(\frac{\omega}{(2-\lambda) \omega+\pi}-\frac{\omega}{(2-\lambda) \omega-\pi}\right) \\
& \times\left(C_{2}\left(\lambda-2 \lambda^{2}\right)(1+\cos ((2-\lambda) \omega))-C_{1}\left(\lambda-2 \lambda^{2}\right) \sin ((2-\lambda) \omega)\right) .
\end{aligned}
$$

We remark that the values $\lambda_{1}$ and $\lambda_{2}$ are from the equation

$$
\lambda^{2} \sin ^{2}(\omega)=\sin ^{2}(\lambda \omega)
$$

and it is not possible to solve directly. So, we use the numerical root-finding method and bisection method for various tolerances of approximated $\lambda$. For internal re-entrant corner angle $\omega$, we choose 400 angles from $\pi$ to $2 \pi$ by $0.0025 \pi$ interval. Denote that

(i) $U_{A} V_{A}:=$ left side of equation (40)

(ii) $U_{A} V_{B}:=$ left side of equation (41) (iii) $U_{B} V_{A}:=$ left side of equation (42)

(iv) $U_{B} V_{B}:=$ left side of equation (43)

We choose three tolerances $1 e^{-12}, 1 e^{-13}$, and $1 e^{-14}$ for solving $\lambda_{1}$ and $\lambda_{2}$. By substituting $\lambda_{1}, \lambda_{2}$, and $\omega$ to (44)-(47). Figure 2 shows numerical substitution values of $U_{A} V_{A}$, $U_{A} V_{B}, U_{B} V_{A}$, and $U_{B} V_{B}$. We can see that the values strongly depend on the tolerances. If $\lambda_{1}$ and $\lambda_{2}$ are exact real values, we can claim our assertion.

Now, we construct the Uzawa method for two singular solutions. We assume that the form (33) is valid. The weak formulation of Step 1 in the Uzawa method test by $\eta_{2 \rho} \mathbf{S}_{L}$ is in the form

$$
\begin{aligned}
& \mu\left\langle\mathbf{w}^{n+1},-\Delta \eta_{2 \rho} \mathbf{S}_{L}^{d}\right\rangle+\left\langle\nabla q^{n}, \eta_{2 \rho} \mathbf{S}_{L}^{d}\right\rangle \\
& \quad+\alpha_{1}^{n+1}\left\langle-\Delta \eta_{\rho} \mathbf{u}_{1}^{s}+\nabla \eta_{\rho} p_{1}^{s}, \eta_{2 \rho} \mathbf{S}_{L}^{d}\right\rangle+\alpha_{2}^{n+1}\left\langle-\Delta \eta_{\rho} \mathbf{u}_{2}^{s}+\nabla \eta_{\rho} p_{2}^{s}, \eta_{2 \rho} \mathbf{S}_{L}^{d}\right\rangle \\
& =\left\langle\mathbf{f}, \eta_{2 \rho} \mathbf{S}_{L}^{d}\right\rangle .
\end{aligned}
$$

Let $\mathbf{w}=\left(w_{1}, w_{2}\right)$ and $\mathbf{f}=\left(f_{1}, f_{2}\right)$. Then, $x$ component of (49) is

$$
\begin{aligned}
\mu\left\langle w_{1}^{n+1},-\Delta \eta_{2 \rho} s_{L}^{d}\right\rangle+\left\langle\left(q^{n}\right)_{x}, \eta_{2 \rho} s_{L}^{d}\right\rangle & \\
& +\alpha_{1}^{n+1}\left\langle-\Delta \eta_{\rho} u_{1}^{s}+\left(\eta_{\rho} p_{1}^{s}\right)_{x}, \eta_{2 \rho} s_{L}^{d}\right\rangle \\
& +\alpha_{2}^{n+1}\left\langle-\Delta \eta_{\rho} u_{2}^{s}+\left(\eta_{\rho} p_{2}^{s}\right)_{x}, \eta_{2 \rho} s_{L}^{d}\right\rangle \\
= & \left\langle f_{1}, \eta_{2 \rho} s_{L}^{d}\right\rangle,
\end{aligned}
$$

and $y$ component of (49) is

$$
\begin{aligned}
\mu\left\langle w_{2}^{n+1},-\Delta \eta_{2 \rho} s_{L}^{d}+\left(q^{n}\right)_{y}, \eta_{2 \rho} s_{L}^{d}\right\rangle & \\
& +\alpha_{1}^{n+1}\left\langle-\Delta \eta_{\rho} v_{1}^{s}+\left(\eta_{\rho} p_{1}^{s}\right)_{y}, \eta_{2 \rho} s_{L}^{d}\right\rangle \\
& +\alpha_{2}^{n+1}\left\langle-\Delta \eta_{\rho} v_{2}^{s}+\left(\eta_{\rho} p_{2}^{s}\right)_{y}, \eta_{2 \rho} s_{L}^{d}\right\rangle \\
= & \left\langle f_{2}, \eta_{2 \rho} s_{L}^{d}\right\rangle .
\end{aligned}
$$



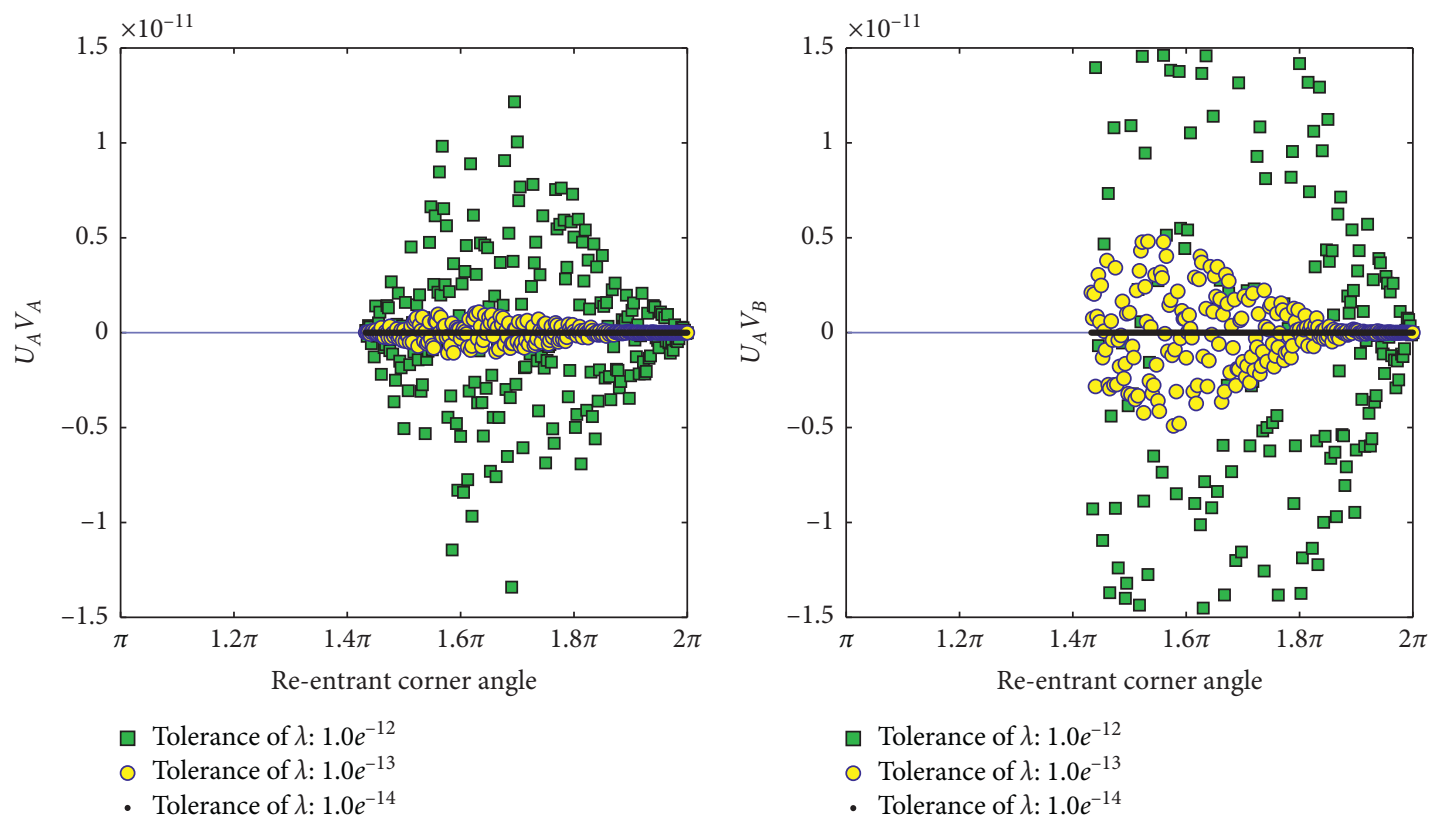

(a)

(b)

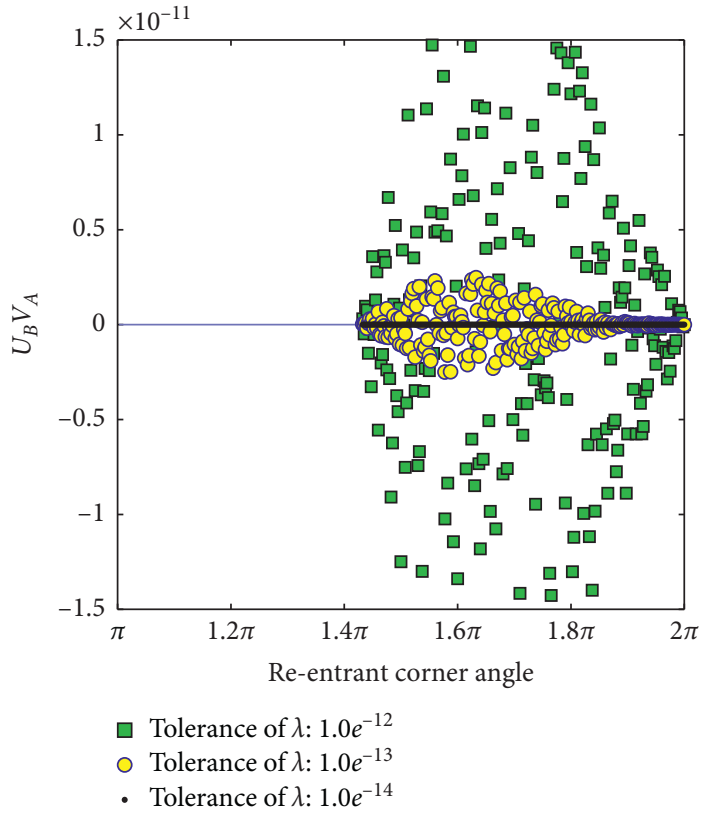

(c)

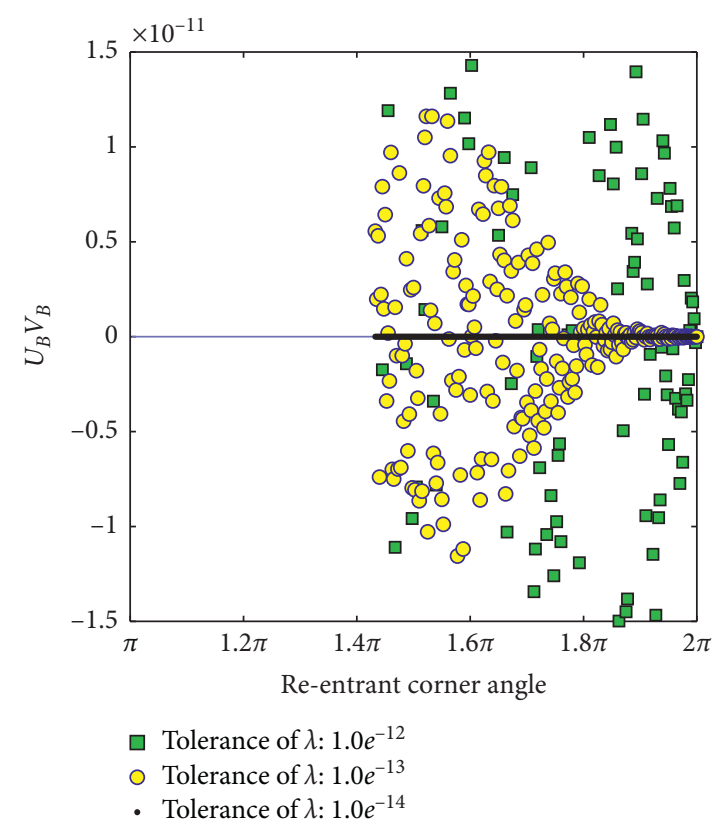

(d)

Figure 2: $U_{A} V_{A}, U_{A} V_{B}, U_{B} V_{A}$, and $U_{B} V_{B}$ values by numerical substitution with various tolerance of $\lambda$. 
If we use the following notations

$$
\begin{aligned}
U s_{1} & =\left\langle-\Delta \eta_{\rho} u_{1}^{s}+\left(\eta_{\rho} p_{1}^{s}\right)_{x}, \eta_{2 \rho} s_{L}^{d}\right\rangle, \\
U s_{2} & =\left\langle-\Delta \eta_{\rho} u_{2}^{s}+\left(\eta_{\rho} p_{2}^{s}\right)_{x}, \eta_{2 \rho} s_{L}^{d}\right\rangle, \\
V s_{1} & =\left\langle-\Delta \eta_{\rho} v_{1}^{s}+\left(\eta_{\rho} p_{1}^{s}\right)_{y}, \eta_{2 \rho} s_{L}^{d}\right\rangle, \\
V s_{2} & =\left\langle-\Delta \eta_{\rho} v_{2}^{s}+\left(\eta_{\rho} p_{2}^{s}\right)_{y}, \eta_{2 \rho} s_{L}^{d}\right\rangle, \\
\beta W_{1}^{n+1} & =\mu\left\langle w_{1}^{n+1},-\Delta \eta_{2 \rho} s_{L}^{d}\right\rangle+\left\langle\left(q^{n}\right)_{x}, \eta_{2 \rho} s_{L}^{d}\right\rangle, \\
\beta W_{2}^{n+1} & =\mu\left\langle w_{2}^{n+1},-\Delta \eta_{2 \rho} s_{L}^{d}\right\rangle+\left\langle\left(q^{n}\right)_{y}, \eta_{2 \rho} s_{L}^{d}\right\rangle, \\
\beta f_{1} & =\left\langle f_{1}, \eta_{2 \rho} s_{L}^{d}\right\rangle, \\
\beta f_{2} & =\left\langle f_{2}, \eta_{2 \rho} s_{L}^{d}\right\rangle,
\end{aligned}
$$

then (50) and (51) can be rewritten by

$$
\begin{aligned}
& \beta W_{1}^{n+1}+\alpha_{1}^{n+1} U s_{1}+\alpha_{2}^{n+1} U s_{2}=\beta f_{1} \\
& \beta W_{2}^{n+1}+\alpha_{1}^{n+1} V s_{1}+\alpha_{2}^{n+1} V s_{2}=\beta f_{2} .
\end{aligned}
$$

Therefore, we obtain

$$
\begin{aligned}
& \alpha_{1}^{n+1} U s_{1} \cdot U s_{1}=\left(\beta f_{1}-\beta W_{1}^{n+1}-\alpha_{2}^{n+1} U s_{2}\right) U s_{1}, \\
& \alpha_{1}^{n+1} V s_{1} \cdot V s_{1}=\left(\beta f_{2}-\beta W_{2}^{n+1}-\alpha_{2}^{n+1} V s_{2}\right) V s_{1} .
\end{aligned}
$$

Finally, combining (54) and (55) and equation (35) in Theorem 2 yields

$$
\begin{aligned}
\alpha_{1}^{n+1} & \left(U s_{1} \cdot U s_{1}+V s_{1} \cdot V s_{1}\right) \\
= & \left(\beta f_{1}-\beta W_{1}^{n+1}\right) U s_{1}+\left(\beta f_{2}-\beta W_{2}^{n+1}\right) V s_{1} \\
& -\alpha_{2}^{n+1}\left(U s_{2} U s_{1}+V s_{2} V s_{1}\right) \\
= & \left(\beta f_{1}-\beta W_{1}^{n+1}\right) U s_{1}+\left(\beta f_{2}-\beta W_{2}^{n+1}\right) V s_{1} .
\end{aligned}
$$

As a result, we can calculate one stress intensity factor $\alpha_{1}^{n+1}$ by

$$
\alpha_{1}^{n+1}=\frac{\left(\beta f_{1}-\beta W_{1}^{n+1}\right) U s_{1}+\left(\beta f_{2}-\beta W_{2}^{n+1}\right) V s_{1}}{U s_{1} \cdot U s_{1}+V s_{1} \cdot V s_{1}} .
$$

Similarly, another stress intensity factor $\alpha_{2}^{n+1}$ can be computed by

$$
\alpha_{2}^{n+1}=\frac{\left(\beta f_{1}-\beta W_{1}^{n+1}\right) U s_{2}+\left(\beta f_{2}-\beta W_{2}^{n+1}\right) V s_{2}}{U s_{2} \cdot U s_{2}+V s_{2} \cdot V s_{2}} .
$$

Remark 2. Since $\left\langle\eta_{\rho} u^{s},-\Delta \eta_{2 \rho} s_{L}^{d}\right\rangle=0$, Lemma 4 is valid for two singular functions case.

Algorithm 3 is the proposed Uzawa iteration for two singularities.

\section{Numerical Experiments}

In this section, we provide numerical simulations using the standard Uzawa method and Algorithm 3. Our goal is to check their performance for the numerical solution $\mathbf{u}_{h}$ and $p_{h}$, which is composed of a regular solution $\mathbf{w}_{h}$ and $q_{h}$ and stress intensity factors $\alpha_{1 h}$ and $\alpha_{2 h}$. All numerical integration is calculated by the Gaussian quadrature 6 points. We notate $\Gamma_{\text {out }}=\{(r, \theta) \in \partial \Omega: \theta \neq 0$ and $\theta \neq \omega\}$.

\section{Example 1. Simulation environments are}

(1) PDE model: Stokes equation (3)

(2) Computational domain: $([-1,1] \times[-1,1]) \backslash([0,1] \times$ $[-1,0]) \in \mathbb{R} \times \mathbb{R}(\Gamma$ shape domain, $\omega=3 \pi / 2)$ (see Figure 3).

(3) Parameters:

$$
\begin{aligned}
& \mu=1.0 \\
& \lambda_{1}=0.5444837367824639 \\
& \lambda_{2}=0.9085291898460987 \\
& \rho=1.0 \text { and radius } R=3.0 \in \mathbb{R} \text { for cut-off function } \\
& \left\|\mathbf{u}^{n+1}-\mathbf{u}^{n}\right\| \leq \text { tolerance }=1.0^{-8}
\end{aligned}
$$

(4) Exact solution (see Figures 4 and 5):

$$
\begin{aligned}
& u=-\sin ^{2}(\pi x) \sin (2 \pi y)+\alpha_{1} u_{1}^{s}+\alpha_{2} u_{2}^{s} \\
& v=\sin (2 \pi x) \sin ^{2}(\pi y)+\alpha_{1} v_{1}^{s}+\alpha_{2} v_{2}^{s} \\
& p=(2+\cos (\pi x))(2+\cos (\pi y))-4+\alpha_{1} p_{1}^{s}+\alpha_{2} p_{2}^{s} \\
& \alpha_{1}=2.0, \alpha_{2}=-3.0
\end{aligned}
$$

(5) Boundary condition: Dirichlet boundary condition. $\mathbf{u}=0$ on $\left.\Gamma_{\text {in }}\right|_{\theta=0}$ and $\left.\Gamma_{\text {in }}\right|_{\theta=\omega}$, and $\mathbf{u} \neq 0$ on $\Gamma_{\text {out }}$.

(6) Finite elements: Taylor-Hood and minielements.

Minielement chooses $\mathbb{V}_{h}$ and $\mathbb{P}_{h}$ are the piecewise P1bubble and piecewise P1 space pair satisfying discrete infsup condition. In this finite dimensional space, we desire $\left\|\mathbf{u}-\mathbf{u}_{h}\right\|_{0} \leq C h^{2}, \quad\left\|I \mathbf{u}-\mathbf{u}_{h}\right\|_{\infty} \leq C h^{2}, \quad\left\|\mathbf{u}-\mathbf{u}_{h}\right\|_{1} \leq C h, \quad$ and $\left\|p-p_{h}\right\|_{0} \leq C h$ as an optimal convergence for some constant $C$ which does not depend on numerical solution $(\mathbf{u}, p)$. In Figure 6, standard Uzawa iteration loses accuracy of $\mathbf{u}$ and $p$ because the solutions contain singular functions. On the contrary, proposed Uzawa algorithm shows optimal accuracy of $\mathbf{w}, q, \alpha_{1}$, and $\alpha_{2}$. Since $\mathbf{u}$ only depends $\mathbf{w}, \alpha_{1}$, and $\alpha_{2}$ and similarly $p$ only depends $q, \alpha_{1}$, and $\alpha_{2}$. The figure guarantees convergence rate of $\mathbf{u}$ and $p$. The errors of stress intensity factors $\alpha_{1}$ and $\alpha_{2}$ decrease somewhat irregularly. However, we can see that the overall decay of both errors follows the optimal rate.

The next finite element space is the Taylor-Hood space which chooses $\mathbb{V}_{h}$ and $\mathbb{P}_{h}$ as the piecewise P2 and piecewise $\mathrm{P} 1$ space pair. This also satisfies the discrete inf-sup condition. In this space, the desired convergence rates are $\left\|\mathbf{u}-\mathbf{u}_{h}\right\|_{0} \leq C h^{3},\left\|I \mathbf{u}-\mathbf{u}_{h}\right\|_{\infty} \leq C h^{3},\left\|\mathbf{u}-\mathbf{u}_{h}\right\|_{1} \leq$ $C h^{2}$, and $\left\|p-p_{h}\right\|_{0} \leq C h^{2}$, and Figure 7 shows the results calculated by each method. Even in this case, standard Uzawa iteration shows insufficient performance, which is 
$\mu\left\langle\nabla \mathbf{z}_{1}, \nabla \mathbf{v}_{h}\right\rangle+-\mu\left\langle\Delta \eta_{\rho} \mathbf{u}_{1}^{s}+\nabla \eta_{\rho} p_{1}^{s}, \mathbf{v}_{h}\right\rangle=0$

$\mu\left\langle\nabla \mathbf{z}_{2}, \nabla \mathbf{v}_{h}\right\rangle+\left\langle-\mu \Delta \eta_{\rho} \mathbf{u}_{2}^{s}+\nabla \eta_{\rho} p_{2}^{s}, \mathbf{v}_{h}\right\rangle=0$

Repeat the following steps until $\left\|\mathbf{w}_{h}^{n+1}-\mathbf{w}_{h}^{n}\right\| \leq$ tolerance:

Step 1: for all $\mathbf{v}_{h} \in \mathbb{W}_{h}$, find $\widehat{w}_{h}^{n+1} \in \mathbb{W}_{h}$ as the solution of $\mu\left\langle\nabla \widehat{w}_{h}^{n+1}, \nabla \mathbf{v}_{h}\right\rangle+\left\langle\nabla q_{h}^{n}, \nabla \mathbf{v}_{h}\right\rangle+\alpha_{1 h}^{n}\left\langle-\Delta \eta_{\rho} \mathbf{u}_{1}^{s}+\nabla\right.$ $\left.\eta_{\rho} p_{1}^{s}, \mathbf{v}_{h}\right\rangle+\alpha_{2 h}^{n}\left\langle-\Delta \eta_{\rho} \mathbf{u}_{2}^{s}+\nabla \eta_{\rho} p_{2}^{s}, \mathbf{v}_{h}\right\rangle=\left\langle\mathbf{f}, \mathbf{v}_{h}\right\rangle$

Step 2: by $\widehat{w}_{h}^{n+1}=\left(W_{1}^{n+1}, W_{2}^{n+1}\right)$, find $\alpha_{1 h}^{n+1}$ and $\alpha_{2 h}^{n+1}$ from the equations $\alpha_{1 h}^{n+1}=\left(\left(\beta f_{1}-\beta W_{1}^{n+1}\right) U s_{1}+\left(\beta f_{2}-\beta W_{2}^{n+1}\right) V s_{1} / U s_{1} \cdot U s_{1}+V s_{1} \cdot V s_{1}\right)$ $\alpha_{2}^{n+1}=\left(\left(\beta f_{1}-\beta W_{1}^{n+1}\right) U s_{2}+\left(\beta f_{2}-\beta W_{2}^{n+1}\right) V s_{2} / U s_{2} \cdot U s_{2}+V s_{2} \cdot V s_{2}\right)$

Step 3: update $\mathbf{w}_{h}^{n+1} \in \mathbb{W}_{h}$, by $\mathbf{w}_{h}^{n+1}=\widehat{w}_{h}^{n+1}+\left(\alpha_{1 h}^{n+1}-\alpha_{1 h}^{n}\right) \mathbf{z}_{1}+\left(\alpha_{2 h}^{n+1}-\alpha_{2 h}^{n}\right) \mathbf{z}_{2}$

Step 4: for all $p_{h} \in \mathbb{P}_{h}$, update $q_{h}^{n+1}$ by $\left\langle q_{h}^{n+1}, p_{h}\right\rangle=\left\langle q_{h}^{n}, p_{h}\right\rangle-\mu\left\langle\nabla \cdot \mathbf{w}_{h}^{n+1}, p_{h}\right\rangle-\alpha_{1 h}^{n+1}\left\langle\nabla \cdot \eta_{\rho} \mathbf{u}_{1}^{s}, p_{h}\right\rangle-\alpha_{2 h}^{n+1}\left\langle\nabla \cdot \eta_{\rho} \mathbf{u}_{2}^{s}, p_{h}\right\rangle$

Algorithm 3: (FE-DSFM Uzawa iteration for two singular functions). Start with any initial values $p_{h}^{0}, \alpha_{1 h}^{0}$, and $\alpha_{2 h}^{0}$. $\mathbf{z}_{1}$ and $\mathbf{z}_{2}$, for all $\mathbf{v}_{h} \in \mathbb{W}_{h}$, is the solution of

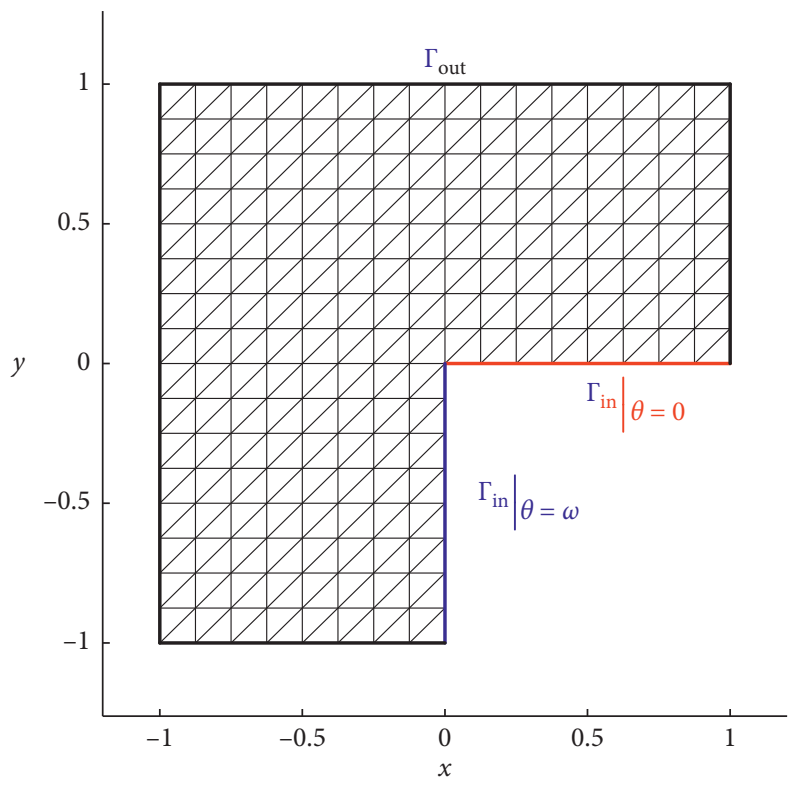

FIgURE 3: Computational domain $(h=1 / 8)$ of Example 1.

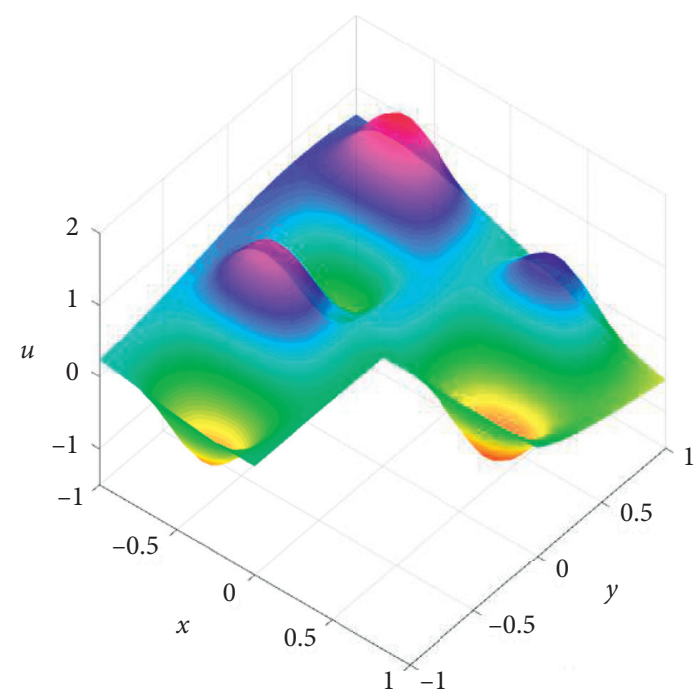

(a)

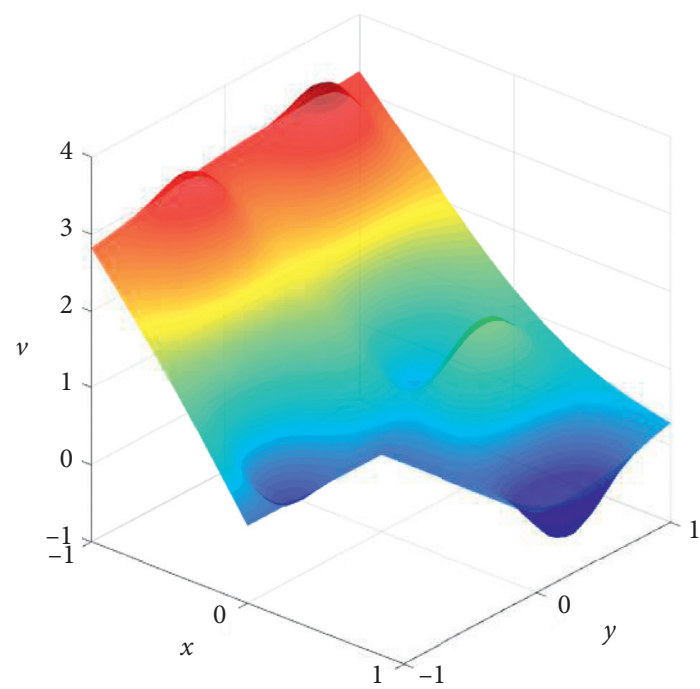

(b)

Figure 4: Exact solutions, $u$ (a) and $v$ (b), of Example 1. 


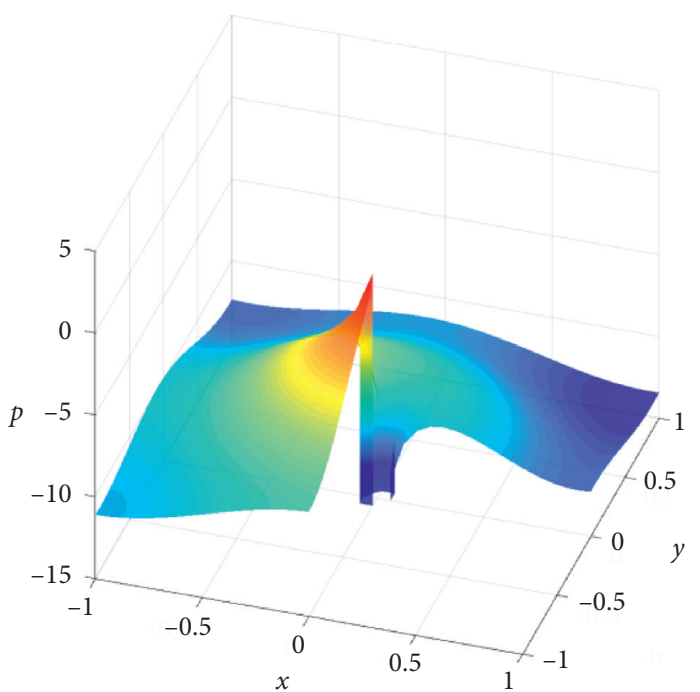

(a)

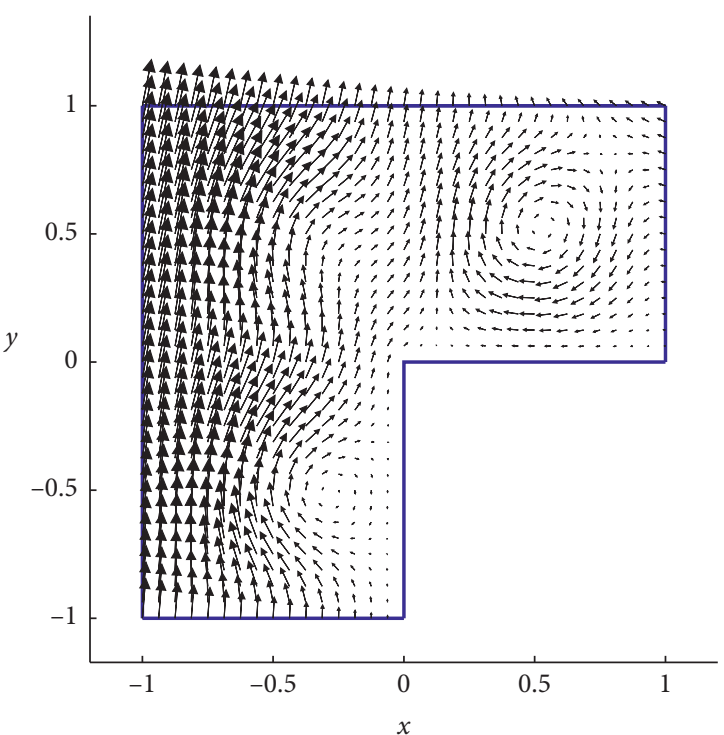

(b)

Figure 5: Exact solution, $p$ (a) and the quiver of $\mathbf{u}(\mathrm{b})$, of Example 1 .

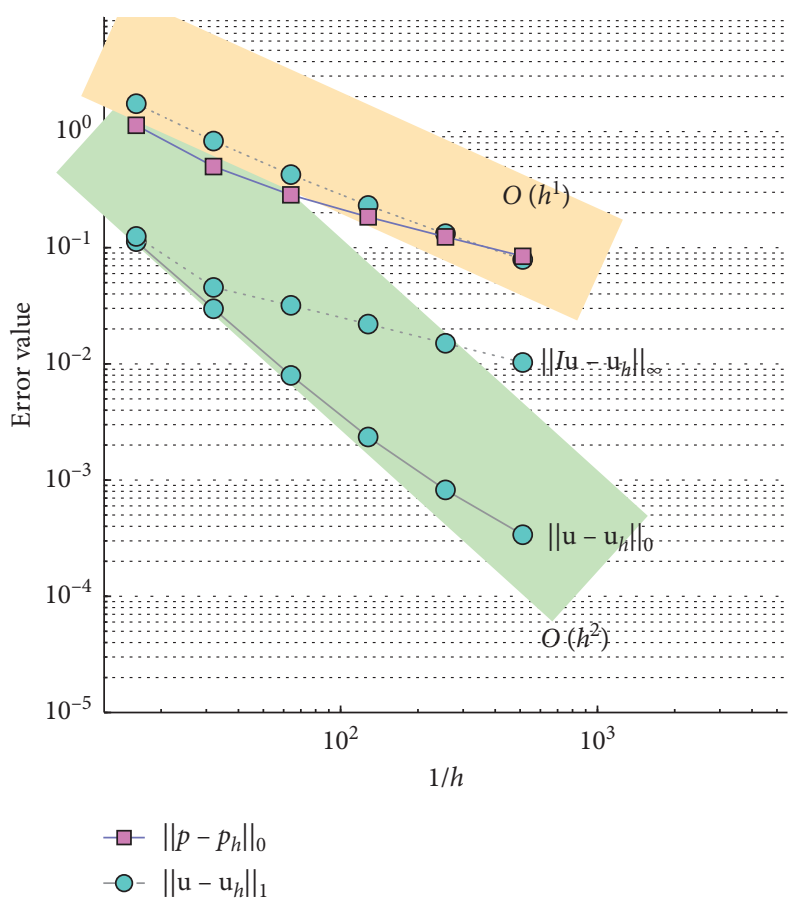

(a)

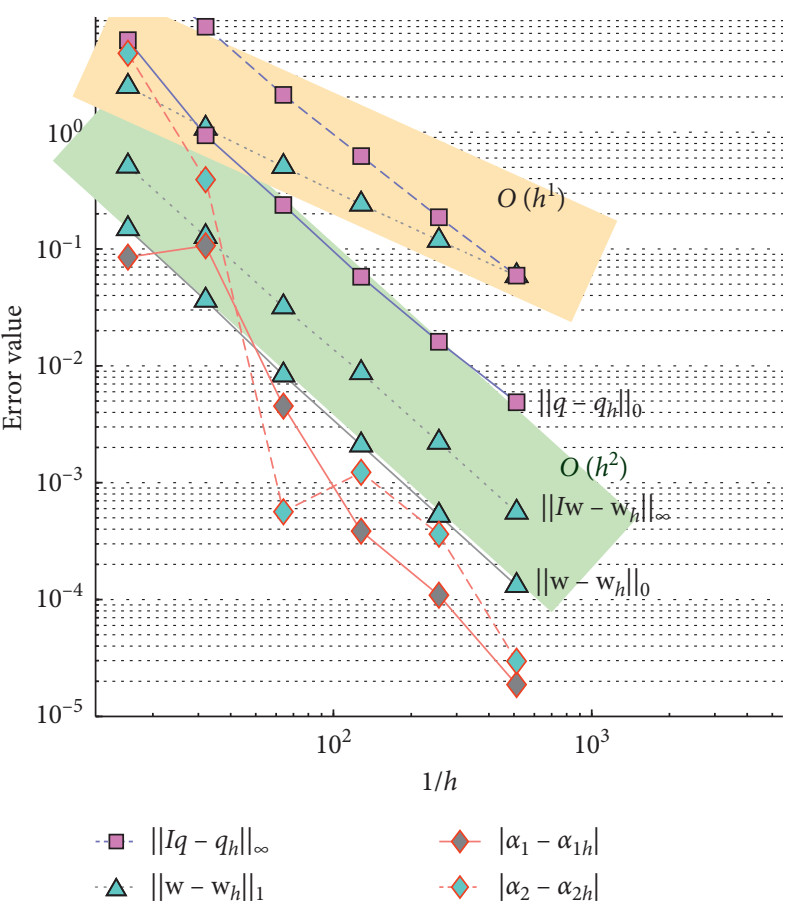

(b)

Figure 6: Error decays between standard Uzawa method and Algorithm 3 for Example 1. All algorithms use minielements. 


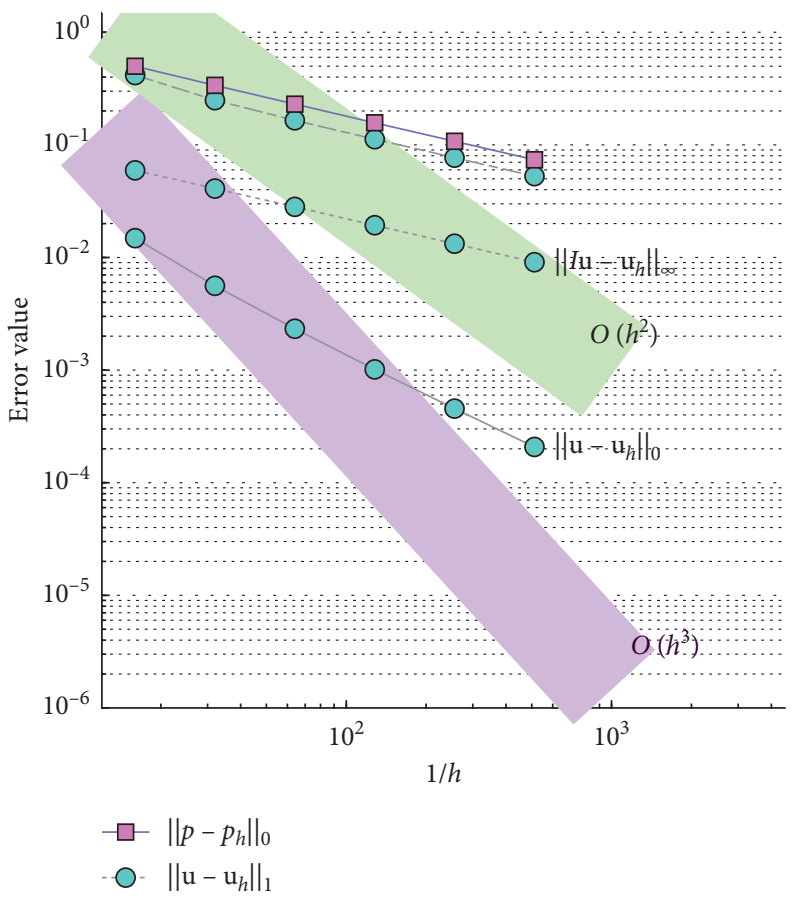

(a)

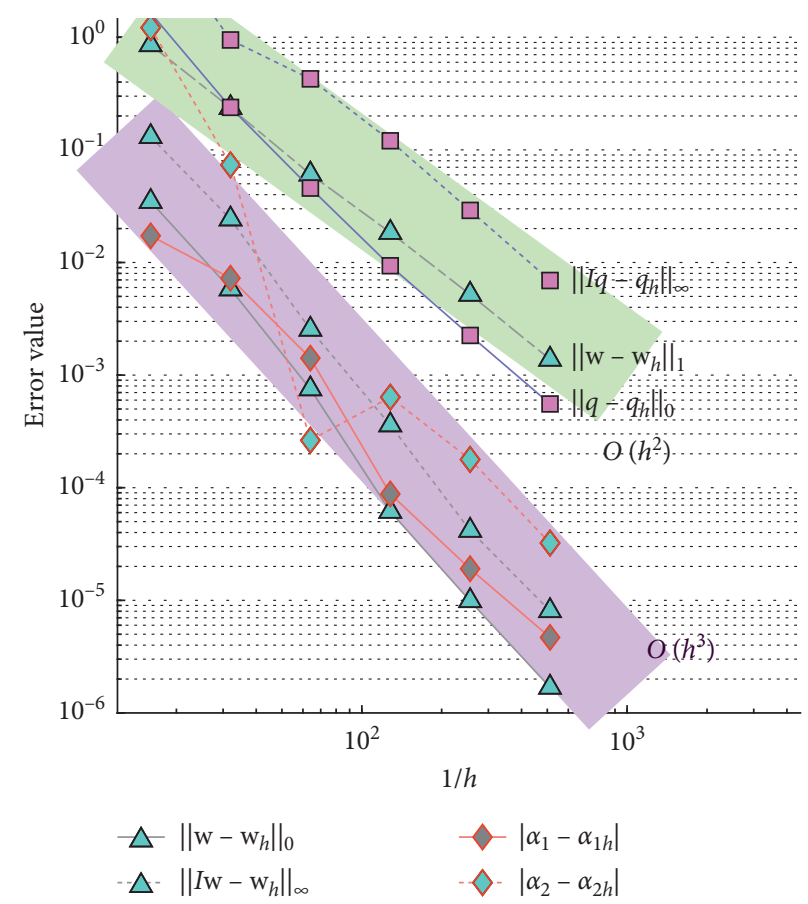

(b)

Figure 7: Error decays between standard Uzawa method and Algorithm 3 for Example 1. All algorithms use Taylor-Hood elements.

almost the similar convergence rate as in the minielement case, despite higher-order finite element space. In contrast, the proposed Uzawa algorithm presents optimal results.

\section{Conclusions}

We have presented a finite element using dual singular functions with Uzawa iteration for the steady Stokes equations including corner singularities. The proposed method uses the fact that the solution of an elliptic boundary value problem can be decomposed of a finite number of singular functions and a smoother remainder function which is called a regular solution. For the decoupled variational formulations with Uzawa iteration, we test the dual singular function of the Laplace problem and another cut-off function. Also, orthogonality of singular function of Stokes equation was presented, which imply that the proposed algorithm is available for two singular functions in the same corner. A numerical example indicates that the scheme is optimally accurate in both velocity and pressure. Besides, the scheme requires only one additional linear solver in the initial step, so it is much more cost-effective than the conventional dual singular function method for solving the Sherman-Morrison formulation. Since our method is based on the Uzawa iteration, it can be an extended version to solve the Navier-Stokes equation later.

\section{Data Availability}

No data were used to support the findings of the study.

\section{Conflicts of Interest}

The authors declare that they have no conflicts of interest.

\section{Acknowledgments}

This research was supported by Basic Science Research Program through the National Research Foundation of Korea (NRF) funded by the Ministry of Education (NRF2020R1A6A3A01097164).

\section{References}

[1] C. Schwab, P-and hp-Finite Element Methods: Theory and Applications in Solid and Fluid Mechanics (Numerical Mathematics and Scientific Computation), Clarendon Press, Oxford, UK, 1999.

[2] I. Babuška and A. Miller, "The post-processing approach in the finite element method-part 1: calculation of displacements, stresses and other higher derivatives of the displacements," International Journal for Numerical Methods in Engineering, vol. 20, pp. 1085-1109, 1984.

[3] I. Babuška and A. Miller, "The post-processing approach in the finite element method-part 2: the calculation of stress intensity factors," International Journal for Numerical Methods in Engineering, vol. 20, pp. 1111-1129, 1984.

[4] B. A. Szabó and Z. Yosibash, "Numerical analysis of singularities in two dimensions part 1: computation of the eigenpairs," International Journal for Numerical Methods in Engineering, vol. 38, pp. 2055-2082, 1995.

[5] B. A. Szabó and Z. Yosibash, "Numerical analysis of singularities in two dimensions part 2: computation of generalized flux/stress intensity factors," International Journal for 
Numerical Methods in Engineering, vol. 39, no. 3, pp. 409-434, 1996.

[6] S. C. Brenner, "Multigrid methods for the computation of singular solutions and stress intensity factors I: corner singularities," Mathematics of Computation, vol. 68, no. 226, pp. 559-584, 1999.

[7] G. C. Georgiou, L. Olson, and Y. S. Smyrlis, "A singular function boundary integral method for the Laplace equation," Communications in Numerical Methods in Engineering, vol. 12, no. 2, pp. 127-134, 1996.

[8] G. Georgiou, A. Boudouvis, and A. Poullikkas, "Comparison of two methods for the computation of singular solutions in elliptic problems," Journal of Computational and Applied Mathematics, vol. 79, no. 2, pp. 277-287, 1997.

[9] M. Elliotis, G. Georgiou, and C. Xenophontos, "The solution of Laplacian problems over $L$-shaped domains with a singular function boundary integral method," Communications in Numerical Methods in Engineering, vol. 18, no. 3, pp. 213-222, 2002.

[10] V. A. Kondratév, "Boundary problems for elliptic equations in domains with conical or angular points," Transactions of the Moscow Mathematical Society, vol. 16, pp. 209-292, 1967.

[11] S. A. Nazarov and B. A. Plamenevsky, Elliptic Problems in Domains with Piecewise Smooth Boundaries, Walter de Gruyter, Berlin, Germany, 1994.

[12] V. A. Kozlov, V. G. Mázya, and J. Rossmann, Elliptic Boundary Value Problems in Domains with Point Singularities, Amer. Math. Soc., Providence, RI, USA, 1997.

[13] G. J. Fix, S. Gulati, and G. I. Wakoff, "On the use of singular functions with finite element approximations," Journal of Computational Physics, vol. 13, no. 2, pp. 209-228, 1973.

[14] E. P. Stephan and W. L. Wendland, "An augmented Galerkin procedure for the boundary integral method applied to twodimensional screen and crack problems," Applicable Analysis, vol. 18, no. 3, pp. 183-219, 1984.

[15] E. Stephan and W. L. Wendland, "An augmented Galerkin procedure for the boundary integral method applied to mixed boundary value problems," Applied Numerical Mathematics, vol. 1, no. 2, pp. 121-143, 1985.

[16] M. Dobrowolski, Numerical Approximation of Elliptic Interface and Corner Problems, Habilitation-schrift, Rheinischen Friedrich-Wilhelms-Universitt, Bonn, Germany, 1981.

[17] H. Blum and M. Dobrowolski, "On finite element methods for elliptic equations on domains with corners," Computing, vol. 28 , no. 1 , pp. 53-63, 1982.

[18] M. Bourlard, M. Dauge, M.-S. Lubuma, and S. Nicaise, "Coefficients of the singularities for elliptic boundary value problems on domains with conical points. III: finite element methods on polygonal domains," SIAM Journal on Numerical Analysis, vol. 29, no. 1, pp. 136-155, 1992.

[19] Z. Cai and S. Kim, "A finite element method using singular functions for the Poisson equation: corner singularities," SIAM Journal on Numerical Analysis, vol. 39, no. 1, pp. 286-299, 2001.

[20] Z. Cai, S. Kim, S. Kim, and S. Kong, "A finite element method using singular functions for Poisson equations: mixed boundary conditions," Computer Methods in Applied Mechanics and Engineering, vol. 195, no. 19-22, pp. 2635-2648, 2006.

[21] S. Kim, Z. Cai, J.-H. Pyo, and S. Kong, "A finite element method using singular functions: interface problems," Hokkaido Mathematical Journal, vol. 36, pp. 815-836, 2007.

[22] S. Kim, J.-H. Pyo, and J. S. Lee, "A finite element method using singular functions for Helmholtz equations: part I," Journal of the Korea Society for Industrial and Applied Mathematics, vol. 12, pp. 13-23, 2008.

[23] D.-K. Jang and J.-H. Pyo, "Finite element dual singular function methods for Helmholtz and heat equations," Journal of the Korea Society for Industrial and Applied Mathematics, vol. 22, no. 2, pp. 101-113, 2018.

[24] Y. N. Anjam, "Singularities and regularity of stationary Stokes and Navier-Stokes equations on polygonal domains and their treatments," American Institute of Mathematical Sciences Mathematics, vol. 5, no. 1, pp. 440-466, 2019.

[25] B. Li, "An explicit formula for corner singularity expansion of the solutions to the Stokes equations in a polygon," International Journal of Numerical Analysis and Modeling, vol. 17, no. 6, pp. 900-928, 2020.

[26] J.-H. Pyo, "A finite element dual singular function method to solve the Stokes equations including corner singularities," International Journal of Numerical Analysis \& Modeling, vol. 12, pp. 516-535, 2015.

[27] D.-K. Jang and J.-H. Pyo, "Algorithms to apply finite element dual singular function method for the Stokes equations including corner singularities," Journal of the Korea Society for Industrial and Applied Mathematic, vol. 23, no. 2, pp. 115-138, 2019.

[28] D.-K. Jang, A Finite element method using dual singular functions for elliptic boundary value problems including corner singularities, Ph.D. dissertation, Kangwon National University, Chuncheon, Republic of Korea, 2020.

[29] V. Girault and P. A. Raviart, Finite Element Methods for Navier-Stokes Equations, Springer-Verlag, Berlin, Germany, 1986.

[30] F. Brezzi and M. Fortin, Mixed and Hybrid Finite Element Methods, Springer-Verlag, Berlin, Germany, 1991.

[31] R. H. Nochetto and J.-H. Pyo, "Optimal relaxation parameter for the Uzawa method," Numerische Mathematik, vol. 98, no. 4, pp. 695-702, 2004.

[32] C. Wu, Y. Xiao, and P. Li, "Semi-proximal augmented Lagrangian method for sparse estimation of high-dimensional inverse covariance matrices," Journal of Applied and $\mathrm{Nu}$ merical Optimization, vol. 2, no. 2, pp. 155-169, 2020. 